\title{
Managerial Performance Incentives and Firm Risk during Economic Expansions and Recessions*
}

\author{
Tanseli Savaser ${ }^{1}$ and Elif Şişli-Ciamarra ${ }^{2}$ \\ ${ }^{1}$ Bilkent University and ${ }^{2}$ Brandeis University
}

\begin{abstract}
We argue that the relationship between managerial pay-for-performance incentives and risk taking is pro-cyclical. We study the relationship between incentives provided by stock-based compensation and firm risk for US non-financial corporations over the two business cycles between 1992 and 2009. We show that a given level of pay-for-performance incentives results in significantly lower firm risk when the economy is in a downturn. The documented pro-cyclical relationship between incentives and risk taking is consistent with state-dependent risk aversion. Our findings contribute to the literature on the depressive effects of performance incentives on firm risk by documenting the importance of the interaction between performance incentives and risk aversion.
\end{abstract}

JEL classification: G01, G3, M52

\section{Introduction}

A significant portion of executive pay packages are in the form of equity-based compensation, which create pay-for-performance sensitivity and is expected to incentivize managers to exert effort and take actions that increase stock values. However, the relationship between pay-for-performance incentives and firm risk is less clear. Pay-for-performance could induce more risk taking because risky projects generally create more value and therefore increase the expected value of incentive compensation. However, it could also induce less risk taking because of a desire to limit portfolio risk. This is mainly because managers are

* We are indebted for useful comments and suggestions to the anonymous referee, Daniel Bergstresser, William Greene, Iftekhar Hassan, Abigail Hornstein, Jens Hilscher, Lubomir Litov, Catherine Mann, Debarshi Nandy, Carol Osler, Oguzhan Ozbas, Alon Raviv, David Yermack, Dan Zhang as well as seminar participants at Brandeis University, Williams College, Northeastern University, 2012 EFA Annual Meeting in Boston, the conference on Teaching Finance at Turbulent Times at Heilbronn University, 2014 Financial Management Association Meetings, 2014 Bilkent Turkish Finance Workshop, and the XXIII International Tor Vergata Conference on Money, Banking, and Finance. 
inherently more risk averse than diversified shareholders due to their organization-specific human capital and/or undiversified wealth portfolios (Amihud and Lev, 1981; Smith and Stulz, 1985; Tufano, 1996).

Despite the well-developed theoretical literature stressing the depressive effects of managerial performance incentives on risk taking, the number of empirical papers testing this argument remains limited. Although existing studies have focused primarily on the variation in incentive compensation, it is a combination of incentive compensation and managerial risk aversion that should impact the relationship between performance incentives and firm risk. In this article, we test the theory exploiting the variation in both incentive compensation and managerial risk aversion. There is growing evidence illustrating that the individual risk aversion coefficients increase during recessions (Cohn et al., 2015; Guiso, Sapienza, and Zingales, 2014). In addition, managerial wealth is expected to decrease during recessions (Davis and von Wachter, 2011; Farber, 2011; Guvenen, Ozkan, and Song, 2014). Both the increase in risk aversion coefficients and the decrease in managerial wealth are expected to translate into lower risk appetite for a given level of performance incentives. Therefore, we propose and test a joint hypothesis that managerial risk aversion increases during recessions and that the increase in risk aversion leads to a weaker relationship between managerial performance incentives and risk taking.

In order to test this hypothesis, we assemble a panel dataset on executive compensation of the chief executive officers (CEOs) of the US public firms between 1992 and 2009, a period that covers two macroeconomic recessions as determined by the National Bureau of Economic Research (NBER). We calculate the CEO performance incentives provided by stock and stock option grants, which amount on average to $37 \%$ of a CEO's pay during our sample period. We measure the sensitivity of a CEO's wealth to firm performance with delta-the change in the dollar value of a CEO's wealth for a one percentage point change in the stock price (Core and Guay, 2002). We also control for risk-taking incentives provided to the managers through stock option compensation. We measure risk-taking incentives by vega-the change in the dollar value of a CEO's wealth for a 0.01 change in the annualized standard deviation of stock returns (Core and Guay, 1999; Coles, Daniel, and Naveen, 2006).

Using the business cycle dates identified by the NBER, we show that the relationship between pay-for-performance incentives and firm risk is positive during economic expansions. However, we observe no significant relationship between pay-for-performance incentives and firm risk during recessions. To state the impact of the recession periods in economic terms, we calculate the effect of increasing a CEO's delta from its 25 th percentile value $(\$ 49,000)$ to its 75 th percentile value $(\$ 403,000)$. Such an increase in CEO incentives is associated with a $16 \%$ increase in firm risk during the expansionary periods, while it has virtually no effect on firm risk during the recession periods. This result is robust to using alternative measures of macroeconomic state as well as to estimation with instrumental variables and simultaneous equation regressions to account for the endogenous nature of compensations contracts (Murphy, 2012).

Anecdotal evidence supports our finding that managers tend to take actions that reduce risk upon entering a recession. The 2009 McKinsey Global Survey has documented that companies responded to the recession by reducing their investments in risky research and development $(\mathrm{R} \& \mathrm{D})$ projects. ${ }^{1}$ Forty-two percent of the survey respondents said their firms

1 "R\&D in the downturn: McKinsey Global Survey Results" available at http://www.mckinsey.com/in sights/operations/r_and_ampd_in_the_downturn_mckinsey_global_survey_results. 
cut $R \& D$ costs, with a large majority shifting their focus to shorter-term and lower-risk projects. The effects of the shift in the R\&D composition were expected to be long-lived-a significant share of executives said that they expected these R\&D cuts to have adverse effects in the coming 3-5 years.

The weaker relationship between pay-for-performance incentives and firm risk during recessions may not be related to the underlying macroeconomic environment per se, but to the general declines in firm values that accompany the recessions. If the impact of macroeconomic state disappears once we control for firm value declines, then the results we present are merely an artifact of decreasing stock values during recessions and would undermine our hypothesis that delta-risk relationship is pro-cyclical. We alleviate this concern by showing that the delta-risk relationship weakens even for firms that experience value increases during recessions. This result indicates that the increase in risk aversion coefficients during recessions (and not the decline in managerial wealth) is the most likely explanation for the pro-cyclicality of the delta-risk relationship.

The ability of the managers to adjust the firm's overall risk profile over a short period of time should vary across firms. Therefore, the paper's main prediction should be more applicable when a manager can alter the firm's risk profile more. Accordingly, we look into the instances when CEOs have more control over firms' resources. We focus on two variables that have been shown to be correlated with managerial control: CEO tenure and product market competition. Using these measures of managerial control, we show that the relationship between firm risk and delta weakens more in recessions for firms that are managed by more powerful CEOs.

One may also argue that during recessions, there would be a mechanical increase in effective financial leverage (therefore in equity risk) as a result of falling stock prices. Even though the paper studies how the sensitivity of equity risk to pay-for-performance sensitivity varies with macroeconomic conditions, and not how equity risk varies with macroeconomic conditions, the effect of financial leverage on equity risk might vary with factors related to pay-for-performance sensitivity. To alleviate this concern, we confirm our findings in a subsample of firms with very low levels of leverage.

We also repeat our analysis using firm-specific risk in order to partially alleviate the concern that the stock return volatility, our measure of firm risk, may be correlated with the macroeconomic state. We use the residuals from the market model to calculate firm-specific risk. In line with our total risk results, we find that the relationship between delta and firmspecific risk weakens as the macroeconomic environment deteriorates.

To summarize, our results provide support for the joint hypothesis that managerial risk aversion increases during recessions and that the increase in risk aversion leads to a weaker delta-firm risk relationship. In particular, we infer from our findings that the same manager with exactly the same level of equity-based compensation facing the same firm characteristics may target a lower (higher) risk level during economic recessions (expansions). The pro-cyclical relationship between incentives and risk taking is consistent with statedependent managerial risk aversion documented in the literature.

Our research contributes to the literature that studies the depressive effects of stockbased compensation on managerial risk taking. Our research also contributes to a small but growing literature on the impact of different economic states on risk-taking behavior. Kempf, Ruenzi, and Thiele (2009) show that mutual fund managers decrease risk during years marked by negative stock market returns, when "employment risk" dominates "compensation incentives". Schoar and Zuo (2013) illustrate that managers who start their 
careers in recessions have more conservative management styles throughout their tenures as CEOs. Similarly, Malmendier and Nagel (2011) find that individuals who have experienced low stock market returns throughout their lives report lower willingness to take financial risks using data from the Survey of Consumer Finances from 1960 to 2007. Finally, DeYoung, Peng, and Yan (2013) show that banks facing stronger (weaker) economic conditions choose more (less) risky business policies. However, none of these studies analyze the link between performance incentives and firm risk under different economic states. We add to this literature by incorporating the role of pay-for-performance incentives. To our knowledge, we provide the first evidence for the pro-cyclicality of the relationship between managerial pay-for-performance incentives and firm risk. Regulators and compensation committees, who design compensation structures may find it useful to consider the procyclical nature of the relationship between performance incentives and firm risk.

\section{Hypothesis Development}

In this article, we argue that a given level of performance incentive would implement a lower (higher) firm risk during economic recessions (expansions). We motivate this hypothesis by augmenting the standard principal-agent model of executive compensation with a state-dependent managerial risk aversion coefficient (Cohn et al., 2015; Guiso, Sapienza, and Zingales, 2014) and state-dependent managerial wealth. In the standard principalagent models, the contracting problem involves risk neutral shareholders designing compensation schemes for risk averse managers. Managers choose effort levels to attain expected utility levels that are at least equal to their outside options, thus satisfying their participation constraints and incentive compatibility constraints. In these models, stockbased compensation naturally emerges as a part of the optimal compensation contract because it incentivizes managers to exert effort and increase stock value by creating pay-forperformance sensitivity (delta). With regards to risk taking, if higher net present value (NPV) projects are also inherently riskier, then higher performance incentives are expected to implement higher firm risk.

However, it has long been recognized that the linear payoff structure of stock may induce a sufficiently risk averse manager to reject risky, positive NPV projects and therefore reduce the riskiness of the firm (Guay, 1999). This is because managers are inherently more risk averse than diversified shareholders due to their organization-specific human capital and/or undiversified wealth portfolios (Amihud and Lev, 1981; Smith and Stulz, 1985; Tufano, 1996). The relationship between performance incentives from stock-based compensation and firm risk is expected to weaken: (i) when managerial wealth declines and/or (ii) when there is an increase in managerial risk aversion coefficient (Ou-Yang, 2003; Dittmann and Yu, 2011; Edmans and Gabaix, 2011).

To alleviate the effect of risk aversion, managers are awarded stock options, which provide risk-taking incentives. As the value of a stock option increases with stock price volatility, option compensation would provide a risk averse manager an incentive to take on risky investments (Jensen and Meckling, 1976; Myers, 1977; Haugen and Senbet, 1981; Smith and Stulz, 1985; Smith and Watts, 1992; Bizjak, Brickley, and Coles, 1993; Gaver and Gaver, 1993; Core and Guay, 1999; Guay, 1999; Coles, Daniel, and Naveen, 2006). Still, option holdings may not be enough to induce managerial risk taking (Guay, 1999; Ju, Leland, and Senbet, 2002) and the CEOs may reduce firm risk even in the presence of 
strong risk-taking incentives at times when managerial risk aversion is more pronounced (Milidonis and Stathopoulos, 2014).

Overall, the theory implies that while more pay-for-performance sensitivity could induce less risk taking because of a desire to limit portfolio risk, it could also encourage more risk taking because risky projects generally create more value and therefore increase the expected value of incentive compensation. Thus, the direction of the relationship between firm risk and pay-for-performance sensitivity can go either way. Two factors that characterize the manager's utility function are critical in determining the relationship: the risk aversion coefficient and the level of wealth.

In this article, we argue that the relationship between firm risk and pay-for-performance sensitivity will be less positive (or more negative) during recessions as risk aversion is expected to increase due to two distinct reasons: First, the risk aversion coefficient is expected to increase during recessions. This increase can be thought of as a shift in the expected utility function of the manager for a given level of wealth. Second, the general decline in stock prices during recessions may lead to a decline in managerial wealth, thereby increasing the CEO's marginal utility of consumption. The wealth effect can be viewed as the manager moving to a more concave region of his/her utility function, making the executive more sensitive to risk. The wealth effect and the risk aversion coefficient channel work in the same direction, implying the joint hypothesis that managerial risk aversion increases during recessions and that the increase in risk aversion leads to a weaker delta-risk relationship.

The empirical literature documents an increase (decrease) in the individual risk aversion coefficients during recessions (expansions). Based on an investor survey and laboratory experiments, Guiso, Sapienza, and Zingales (2014) show that individual risk aversion substantially increased with the onset of the 2008 financial crisis. They attribute the documented increase in risk aversion to the "fear of large losses" because even those survey responders who have not incurred financial losses still exhibited a decline in their risk aversion suggesting that actual wealth decline is not the only factor driving the changes in risk aversion. In another experiment, Cohn et al. (2015) prime financial professionals with boom and bust scenarios and show that subjects who were primed with a financial bust were substantially more risk averse than those who were primed with a financial boom.

Since neither the managerial risk aversion parameter nor the total managerial wealth is observable, we are not able to directly measure the magnitude of the two effects. However, we note that both channels work in the same direction and suggest a joint hypothesis that managerial risk aversion increases during recessions and that the increase in risk aversion leads to a weaker delta-risk relationship. Our empirical tests support this joint hypothesis. In addition, we show that even those firms whose value increase during recessions experience a weakening delta-firm risk relationship. This implies that the state-dependent link between delta and firm risk emerges independent of the wealth effect, as a consequence of the change in the manager's risk aversion coefficient.

\section{Data}

Our sample consists of the US companies that are covered in the Standard and Poor's ExecuComp database between fiscal years 1992 and 2009. We exclude financial and utility firms. We merge the ExecuComp data with financial data from Compustat and with stock price data from Center for Research in Security Prices (CRSP). We require that a firm has 3 years of consecutive financial data and that its stock has been traded for at least 100 days 
in those fiscal years. We convert the all of the dollar-based variables to 1992 dollars using the GDP deflator.

For each CEO, we get compensation data from ExecuComp. The reporting of compensation variables has changed in ExecuComp following the implementation of FAS 123R for fiscal years ending after December 15, 2006. We follow Hayes, Lemmon, and Qiu (2012) to perform the necessary modifications to ExecuComp variables during 2006-09. We measure the sensitivity of a CEO's wealth from her employment to firm performance with delta-the change in the dollar value of a CEO's wealth for a one percentage point change in the stock price. Mean delta is $\$ 531,000$. Similarly, we measure the sensitivity of a CEO's wealth from her employment to firm risk with vega-the change in the dollar value of a CEO's wealth for a 0.01 change in annualized standard deviation of stock returns. Mean vega is $\$ 80,000$. We winsorize delta, vega, bonus, and salary at the 1st and 99th percentiles (Core and Guay, 2002; Coles, Daniel, and Naveen, 2006). Table I provides the summary statistics on the compensation variables and firm financial characteristics.

We measure firm risk using realized stock return volatility, which is estimated as the annualized variance of daily stock returns over a fiscal year (Guay, 1999; Low, 2009; Hayes, Lemmon, and Qiu, 2012). We also calculate firm-specific risk by estimating the market model using CRSP value-weighted returns as our proxy for the returns of the market portfolio. Firm-specific risk is the annualized variance of the residuals. Innovations to a firm's stock returns are reactions to news about the firm's future expected cash flows as a result of its investment and financing activities. Therefore, higher realized stock return volatility should reflect business decisions that have a larger impact on a firm's expected cash flow volatility into the future and provide an adequate measure of firm riskiness. Alternatively, one can infer firm risk from financial and investment policies such as changes in capital expenditures, $R \& D$ expenditures, leverage or firm diversification, which will affect the volatility of the cash flows into the future. However, many aspects of uncertainty regarding these policies are unobservable. Realized stock return volatility represents the net effect of all managerial risk-taking activities, including those that are unobservable, hence is an appropriate way of capturing firm risk (Low, 2009). Yet, one limitation of our firm risk measure is that it focuses on equity risk alone although total firm risk also includes the debt component. Since the data on private and public debt are not readily available, we are unable to estimate the risk of the firm's debt, but as standard in the literature, we do control for the impact of leverage on equity risk.

We use six variables to measure the macroeconomic state. First, we create a recession indicator variable based on the business cycle dates determined by the NBER. The NBER identified two recessions that coincide with our sample period: March 2001-November 2001 and December 2007-June 2009. If a firm's fiscal year coincides with an NBER recession period for at least 90 days, we assign the value one to the recession indicator variable. The NBER recession indicator takes the value one for 4,098 firm years, corresponding to $18 \%$ of our full sample (Table I). As our second measure of the macroeconomic state, we count the number of days in a firm's fiscal year that coincide with the NBER recession dates.

The NBER recession dates may not precisely correspond to the declines in macroeconomic activity. According to the NBER, the "economic activity is typically below normal in the early stages of an expansion and it sometimes remains so well into the expansion." Also, the NBER recession indicator does not capture the degree of macroeconomic downturns or expansions. With these concerns, we repeat our analyses using alternative measures 
Table I. Summary statistics

This table presents the summary statistics for the variables used in the analyses. The definition of the variables and the relevant data sources are provided in Appendix A.

\begin{tabular}{|c|c|c|c|c|c|c|}
\hline & $N$ & Mean & $\begin{array}{l}\text { Standard } \\
\text { deviation }\end{array}$ & $\mathrm{p} 25$ & $\mathrm{p} 50$ & p75 \\
\hline \multicolumn{7}{|l|}{ A. CEO compensation measures } \\
\hline Salary $(\$ 000 s)$ & 22,906 & 484 & 246 & 304 & 440 & 624 \\
\hline Bonus (\$000s) & 22,906 & 524 & 750 & 59 & 281 & 660 \\
\hline Cash compensation $(\$ 000)$ & 22,906 & 1013 & 923 & 437 & 724 & 1259 \\
\hline Vega (\$000s) & 22,906 & 80 & 144 & 7 & 28 & 84 \\
\hline $\log (1+$ Vega $)$ & 22,906 & 3.15 & 1.80 & 2.05 & 3.38 & 4.44 \\
\hline Delta (\$000s) & 22,906 & 531 & 1368 & 49 & 140 & 403 \\
\hline $\log (1+$ Delta $)$ & 22,906 & 4.88 & 2 & 4 & 5 & 6 \\
\hline Tenure as CEO (years) & 22,906 & 8 & 8 & 3 & 6 & 10 \\
\hline Long tenure & 22,906 & 0.71 & 0.45 & 0 & 1 & 1 \\
\hline Delta (\$-\$ sensitivity) & 22,906 & 0.37 & 0.68 & 0.06 & 0.14 & 0.35 \\
\hline Vega ( $\$-\$$ sensitivity) & 22,906 & 0.06 & 0.08 & 0.01 & 0.03 & 0.07 \\
\hline \multicolumn{7}{|l|}{ B. Risk measures } \\
\hline Total risk & 22,906 & 0.276 & 0.366 & 0.092 & 0.171 & 0.331 \\
\hline $\log$ (Total risk) & 22,906 & -1.726 & 0.914 & -2.381 & -1.764 & -1.105 \\
\hline Idiosyncratic risk & 22,906 & 0.231 & 0.307 & 0.075 & 0.141 & 0.276 \\
\hline $\log$ (Idiosyncratic risk) & 22,906 & -1.924 & 0.934 & -2.590 & -1.961 & -1.289 \\
\hline \multicolumn{7}{|l|}{ C. Firm financial characteristics } \\
\hline Total assets ( $\$ \mathrm{mn})$. & 22,902 & 3,799 & 16,003 & 281 & 735 & 2,235 \\
\hline Net sales ( $\$ \mathrm{mn}$. .) & 22,897 & 3,372 & 10,771 & 284 & 768 & 2,291 \\
\hline Sales growth & 20,515 & 0.07 & 0.27 & -0.03 & 0.06 & 0.16 \\
\hline Market-to-book ratio & 22,891 & 1.85 & 1.51 & 0.95 & 1.37 & 2.14 \\
\hline Leverage ratio & 22,902 & 0.21 & 0.20 & 0.04 & 0.20 & 0.33 \\
\hline$R \& D$ expenditures/assets & 22,902 & 0.04 & 0.07 & 0.00 & 0.00 & 0.05 \\
\hline Capital expenditures/assets & 22,703 & 0.06 & 0.06 & 0.02 & 0.04 & 0.08 \\
\hline FAS cost & 22,897 & 0.07 & 2.84 & 0.00 & 0.00 & 0.02 \\
\hline Surplus cash & 22,635 & 0.08 & 0.11 & 0.03 & 0.08 & 0.13 \\
\hline Cash/assets & 22,897 & 0.20 & 0.23 & 0.03 & 0.10 & 0.29 \\
\hline Return on assets (ROA) & 20,536 & 0.06 & 0.21 & 0.02 & 0.07 & 0.13 \\
\hline Stock return & 20,536 & 0.10 & 1.09 & -0.26 & -0.01 & 0.26 \\
\hline Total loss carry forward & 22,906 & 0.37 & 0.48 & 0 & 0 & 1 \\
\hline Value decline & 22,472 & 0.40 & 0.49 & 0 & 0 & 1 \\
\hline HHI & 22,906 & 324.18 & 354.85 & 127 & 190 & 362 \\
\hline \multicolumn{7}{|l|}{ D. Macroeconomic state measures } \\
\hline Recession dummy & 22,906 & 0.18 & 0.38 & 0 & 0 & 0 \\
\hline Recession days & 22,906 & 46 & 105 & 0 & 0 & 0 \\
\hline GDP growth rate & 22,906 & 0.96 & 2.06 & 0.50 & 1.30 & 2.30 \\
\hline Additions to non-farm payroll & 22,906 & -140 & 201 & -201 & -57 & -4 \\
\hline Personal expenditure growth rate & 22,906 & -0.32 & 0.52 & -0.50 & -0.20 & 0.10 \\
\hline Retail sales growth rate & 22,906 & -1.17 & 0.98 & -1.40 & -0.90 & -0.40 \\
\hline S\&P500 volatility & 22,906 & 0.01 & 0.01 & 0.01 & 0.01 & 0.01 \\
\hline
\end{tabular}


GDP QUARTERLY RELEASE VALUES (\%)

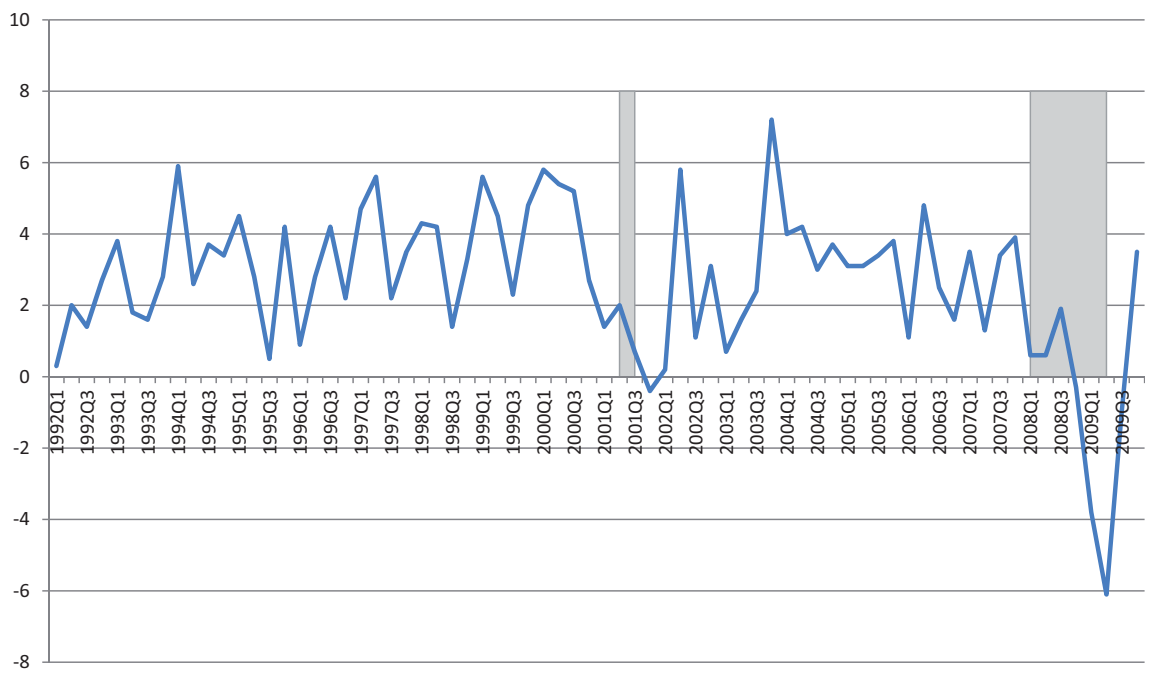

Figure 1. Gray-shaded areas represent the recession periods as identified by the NBER.

of macroeconomic conditions that are based on the advance release values of real GDP, non-farm payroll, personal expenditure, and retail sales growth. Definitions, data sources, and descriptive statistics are presented in Appendix A and Table AI. Earlier literature shows that these macroeconomic indicators have a significant effect on the market sentiment (McQueen and Roley, 1993; Flannery and Protopapadakis, 2002; Boyd, Jagannathan, and $\mathrm{Hu}, 2005$; Andersen et al., 2007). In Figure 1, we plot the advance release values of real GDP growth rates together with the official NBER recession dates. We use the unrevised announcement values of the macroeconomic variables to capture the macroeconomic climate as perceived by the firms during a fiscal year.

\section{Results}

\subsection{Baseline Model}

We use the following empirical specification to test for the relationship between managerial performance incentives and firm risk:

$$
\text { Firm risk } \text { ritt }=\alpha+\beta_{1} \text { Delta }_{i, t-1}+\beta_{2} \operatorname{Vega}_{i, t-1}+\sum_{j} \gamma_{j} X_{i, t}+\varepsilon_{i, t} .
$$

Our primary measure of firm risk is its stock return volatility and main variable of interest is delta. Although pay-for-performance is the primary focus of this article, we also control pay-for-risk sensitivity (vega) that are provided to managers via stock options. This is important because of the possibility that stock options that managers hold may slide out of the money during recessions, diminishing the risk-taking incentives of managers. Firm risk, delta, and vega enter the regressions in their natural logarithm forms. Control variables, $X_{i, t}$, include cash compensation, firm size, market-to-book ratio, research and development expenditures, capital expenditures, and leverage ratio. Coles, Daniel, and Naveen (2006) 
Table II. Performance incentives and risk

This table presents the results for the estimation of Equation (1) in the text. The dependent variable is firm risk, calculated as the logarithm of the annualized variance of the daily stock returns. The main variable of interest is the logarithm of the lagged value of delta, and represents the managerial performance incentives. The definitions of the rest of the variables are provided in Appendix A. All regressions control for year fixed effects. Robust standard errors are clustered at firm level in regressions that control for industry fixed effects and at manager level in regressions that control for firm-manager pair fixed effects. $P$-values are provided in brackets. $*^{* *}$, and $*^{* *}$ mark the $10 \%, 5 \%$, and $1 \%$ statistical significance for the estimated coefficients.

\begin{tabular}{|c|c|c|c|c|c|}
\hline & $\begin{array}{l}\text { Industry } \\
\text { fixed effects } \\
1993-2009\end{array}$ & $\begin{array}{l}\text { Firm fixed } \\
\text { effects } \\
\text { 1993-2009 }\end{array}$ & $\begin{array}{l}\text { Firm-manager } \\
\text { fixed effects } \\
1993-2009\end{array}$ & $\begin{array}{l}\text { Industry fixed } \\
\text { effects } \\
1993-2002\end{array}$ & $\begin{array}{l}\text { Industry fixed } \\
\text { effects } \\
1993-2002\end{array}$ \\
\hline $\log \left(\right.$ Delta $\left._{-1}\right)$ & $\begin{array}{c}-0.000 \\
{[0.963]}\end{array}$ & $\begin{array}{l}0.014 \\
{[0.005]^{* * *}}\end{array}$ & $\begin{array}{c}0.021 \\
{[0.000]^{* * *}}\end{array}$ & $\begin{array}{c}0.042 \\
{[0.000]^{* * *}}\end{array}$ & $\begin{array}{c}0.015 \\
{[0.024]^{* *}}\end{array}$ \\
\hline $\log \left(\operatorname{Vega}_{-1}\right)$ & $\begin{array}{l}-0.032 \\
{[0.000]^{* * *}}\end{array}$ & $\begin{array}{l}-0.051 \\
{[0.000]^{* * *}}\end{array}$ & $\begin{array}{l}-0.047 \\
{[0.000]^{* * *}}\end{array}$ & $\begin{array}{l}0.041 \\
{[0.000]^{* * *}}\end{array}$ & $\begin{array}{c}0.001 \\
{[0.896]}\end{array}$ \\
\hline Log(Cash compensation -1$)$ & $\begin{array}{l}-0.087 \\
{[0.000]^{* * *}}\end{array}$ & $\begin{array}{l}-0.072 \\
{[0.000]^{* * *}}\end{array}$ & $\begin{array}{l}-0.057 \\
{[0.000]^{* * *}}\end{array}$ & $\begin{array}{l}-0.100 \\
{[0.000]^{* * *}}\end{array}$ & $\begin{array}{l}-0.102 \\
{[0.000]^{* * *}}\end{array}$ \\
\hline CEO tenure & $\begin{array}{c}-0.001 \\
{[0.435]}\end{array}$ & $\begin{array}{l}-0.003 \\
{[0.050]^{*}}\end{array}$ & $\begin{array}{c}0.014 \\
{[0.365]}\end{array}$ & $\begin{array}{l}-0.005 \\
{[0.002]^{* * *}}\end{array}$ & $\begin{array}{c}-0.002 \\
{[0.211]}\end{array}$ \\
\hline $\log ($ Sales $)$ & $\begin{array}{l}-0.173 \\
{[0.000]^{* * *}}\end{array}$ & $\begin{array}{l}-0.151 \\
{[0.000]^{* * *}}\end{array}$ & $\begin{array}{l}-0.113 \\
{[0.000]^{* * *}}\end{array}$ & $\begin{array}{l}-0.216 \\
{[0.000]^{* * *}}\end{array}$ & $\begin{array}{l}-0.188 \\
{[0.000]^{* * *}}\end{array}$ \\
\hline Market-to-book & $\begin{array}{c}-0.012 \\
{[0.067]^{*}}\end{array}$ & $\begin{array}{c}0.008 \\
{[0.162]}\end{array}$ & $\begin{array}{c}-0.004 \\
{[0.516]}\end{array}$ & $\begin{array}{l}0.016 \\
{[0.029]^{* *}}\end{array}$ & $\begin{array}{c}0.005 \\
{[0.433]}\end{array}$ \\
\hline $\mathrm{R} \& \mathrm{D}$ expenditures/assets & $\begin{array}{c}1.861 \\
{[0.000]^{* * *}}\end{array}$ & $\begin{array}{c}0.234 \\
{[0.084]^{*}}\end{array}$ & $\begin{array}{c}0.269 \\
{[0.073]^{*}}\end{array}$ & $\begin{array}{l}1.749 \\
{[0.000]^{* * *}}\end{array}$ & $\begin{array}{l}2.030 \\
{[0.000]^{* * *}}\end{array}$ \\
\hline Capital expenditures/assets & $\begin{array}{l}0.337 \\
{[0.034]^{* *}}\end{array}$ & $\begin{array}{c}0.381 \\
{[0.005] * * *}\end{array}$ & $\begin{array}{l}0.400 \\
{[0.009] * * *}\end{array}$ & $\begin{array}{l}-0.908 \\
{[0.000] * * *}\end{array}$ & $\begin{array}{c}0.092 \\
{[0.637]}\end{array}$ \\
\hline Leverage ratio & $\begin{array}{l}0.247 \\
{[0.000]^{* * *}}\end{array}$ & $\begin{array}{l}0.301 \\
{[0.000]^{* * *}}\end{array}$ & $\begin{array}{l}0.402 \\
{[0.000]^{* * *}}\end{array}$ & $\begin{array}{l}0.344 \\
{[0.000]^{* * *}}\end{array}$ & $\begin{array}{l}0.189 \\
{[0.008]^{* * *}}\end{array}$ \\
\hline Constant & $\begin{array}{l}-0.549 \\
{[0.005]^{* * *}}\end{array}$ & $\begin{array}{l}-0.674 \\
{[0.000]^{* * *}}\end{array}$ & $\begin{array}{l}-1.011 \\
{[0.000]^{* * *}}\end{array}$ & $\begin{array}{c}-0.245 \\
{[0.077]^{*}}\end{array}$ & $\begin{array}{l}-0.560 \\
{[0.001]^{* * *}}\end{array}$ \\
\hline$N$ & 20,352 & 20,352 & 18,184 & 20,352 & 20,352 \\
\hline Year fixed effects & Yes & Yes & Yes & No & Yes \\
\hline$R$-squared & 0.549 & 0.513 & 0.532 & 0.378 & 0.564 \\
\hline Adjusted $R$-squared & 0.547 & 0.512 & 0.532 & 0.374 & 0.561 \\
\hline
\end{tabular}

and Armstrong and Vashishtha (2012) provide a detailed discussion of these control variables and their predicted signs in the firm risk equation.

We present the results in Table II. In Column 1, the equation is estimated with industry fixed effects based on firms' two-digit SIC codes, controlling for any time-invariant differences across industries that may contribute to the relationship between CEO incentives and firm risk. In Column 2, we estimate the equation using firm fixed effects, which control for time-invariant differences across firms. Firm fixed effects are used to mitigate the concern that unobservable characteristics might be affecting both the structure of executive compensation and firm risk choices. Finally, in Column 3, we estimate the equation using fixed effects for CEO employment spells. To apply this method, we construct a dummy variable 
for each unique combination of CEO and firm pairs. This specification, as suggested by Graham, Harvey, and Puri (2013) takes into account the possibility that a given CEO might be compensated differently in similar firms due to the heterogeneity in unobservable firm characteristics such as corporate culture. It also alleviates the concern that career concerns and fear of large losses which affect risk aversion might vary for different managers due to the heterogeneity in their unobserved characteristics such as outside employment options. The fixed effects estimations would help alleviate the endogeneity concern that pay-for-performance sensitivity and return volatility might both be correlated with an unobserved firm or managerial characteristic. However, an additional endogeneity concern in the context of our study is that the relation between volatility and pay-for-performance sensitivity might be correlated with some factor related to economic growth. As such, in Section 5.2, we also estimate the instrumental variables regressions that directly deal with the possibility that the interaction of delta and the macroeconomic state may be endogenous with respect to risk taking. All regressions also include year fixed effects to capture systemic variations in firm risk over time. We cluster the standard errors at the firm-year level.

The results indicate a positive relationship between managerial performance incentives and firm risk. Based on the estimation using firm-manager fixed effects, increasing managerial performance incentives from its 25 th percentile $(\$ 49,000)$ to its 75 th percentile $(\$ 403,000)$ is associated with a $15 \%$ increase in firm risk.

Our findings also reveal a statistically significant negative relationship between managerial risk-taking incentives (vega) and firm risk. Earlier studies (e.g., Coles, Daniel, and Naveen, 2006; Low, 2009) find a positive correlation between vega and firm risk. Even though our emphasis in this article is performance incentives (delta), we briefly investigate the reason for the difference in our findings with respect to vega. Since we have followed the specification in Coles, Daniel, and Naveen (2006), we attribute the differences in our results to the different sample periods. In Columns 4 and 5, we estimate the equation for 1992-2002 in order to replicate the analyses in Coles, Daniel, and Naveen (2006) and we obtain a positive coefficient on vega. In a more recent study, Milidonis and Stathopoulos (2014) also report a significant negative relationship between vega and risk and attribute the finding to managerial career concerns. Savaser and Şişli-Ciamarra (2014) attribute the change in the vega-risk relationship to the announcement and implementation of an accounting rule (FAS 123R) that required the expensing of the employee stock options.

\subsection{Macroeconomic State, Incentives, and Firm Risk}

In order to test our main hypothesis that the positive relationship between firm risk and managerial performance incentives becomes weaker (stronger) during macroeconomic downturns (expansions), we augment our baseline model by adding various measures of macroeconomic state and interacting these measures with performance incentives:

Firm risk rit $_{i}=\alpha+\beta_{1}$ Delta $_{i, t-1}+\beta_{2}$ Vega $_{i, t-1}+\delta_{1}$ Macroeconomic State $_{i, t}$.

$$
\begin{aligned}
& +\delta_{2} \text { Macroeconomic State }_{i, t} * \text { Delta }_{i, t-1} \\
& +\delta_{3} \text { Macroeconomic State }_{i, t} * \text { Vega }_{i, t-1} \\
& +\sum_{j} \gamma_{j} X_{i, t}+\varepsilon_{i, t} .
\end{aligned}
$$

The main coefficient of interest belongs to the interaction of the Macroeconomic State with Delta $\left(\delta_{2}\right)$. Our first two measures for the macroeconomic state are based on the NBER 
business cycle dates. We predict a statistically significant negative value for $\delta_{2}$, since we expect managerial risk aversion to increase during recessions.

The results are presented in Table III. In line with the predictions of our hypothesis, we obtain a negative and statistically significant coefficient on the interaction term for all specifications. For example, the interaction coefficient $\left(\delta_{2}\right)$ is -0.023 when we use firmmanager fixed effects (Column 3 ), meaning that a $1 \%$ increase in delta is associated with a $0.023 \%$ decrease in firm risk during a recession. To state the economic impact of recession on the relationship between performance incentives and firm risk, we calculate the impact of an increase in a CEO's delta from its 25 th percentile to its 75 th percentile. Such an increase in performance incentives is associated with a $16 \%$ increase in firm risk during expansions but virtually no increase in risk during recessions. In Columns 4-6 of Table III, we use the actual number of recession days over a firm's fiscal year. The results are similar. Each additional day of recession decreases the effect of delta on firm risk by $0.003 \%$.

We also estimate Equation (2) using the advance release values of growth in seasonally adjusted real GDP, non-farm payroll employment, personal expenditure, and retail sales. Since an increase in these measures corresponds to a better macroeconomic state (thus lower managerial risk aversion), we predict a positive and significant coefficient for their interactions with Delta $\left(\delta_{2}\right)$. In line with the predictions of our hypothesis, we obtain a statistically significant positive coefficient on the interaction term for all specifications (Table IV). For brevity, we focus on the specification using the GDP-based measure and firm-manager fixed effects when discussing our results (Column 3). The coefficient on the interaction term is 0.009 and statistically significant at the $1 \%$ level (Column 3 ). This coefficient suggests that when the economy is at its highest state during our sample period (GDP growth measure $=4.8 \%$ ), an increase in managerial performance incentives from its 25 th percentile to its 75 th percentile would be associated with a $40 \%$ increase in firm risk. On the other hand, when the economy is at its lowest state (GDP growth measure $=-6.1 \%$ ), the same increase in performance incentives would result in a $30 \%$ decline in firm risk. The results using non-farm payroll, personal consumption expenditure, retail sales are presented in the remainder of Table IV and are similar.

To summarize, our findings present evidence that the relationship between equity-based compensation and risk taking depends on the state of the economy. In particular, we infer that the same manager with exactly the same level of performance incentives (delta) facing the same firm characteristics may target a lower (higher) risk level during economic recessions (expansions). The documented pro-cyclical relationship between incentives and risk taking is consistent with state-dependent risk aversion (Guiso, Sapienza, and Zingales, 2014; Cohn et al., 2015) and support our joint hypothesis that managerial risk aversion increases during recessions and that the increase in risk aversion leads to a weaker relationship between managerial performance incentives and risk taking.

\section{Robustness}

\subsection{Firm-Specific Risk}

We repeat our analyses using firm-specific risk as the dependent variable in order to alleviate, at least partially, the concern that the stock return volatility is correlated with the macroeconomic state. As before, the main coefficient of interest belongs to the interaction of the Macroeconomic State and Delta $\left(\delta_{2}\right)$, and we predict a negative (positive) $\delta_{2}$ when we use NBER recessions (macrovariables) as the measure of the macroeconomic state. 
Table III. Relationship between managerial performance incentives and firm risk during macroeconomic recessions

This table presents the results for the estimation of Equation (2) in the text. The dependent variable is firm risk, calculated as the logarithm of the annualized variance of the daily stock returns. The main variable of interest is the interaction of logarithm of the lagged value of delta with the recession measure. The definitions of the variables are provided in Appendix A. All regressions control for year fixed effects. Robust standard errors are clustered at firm level in regressions that control for industry fixed effects and at firm-manager level in regressions that control for firm-manager pair fixed effects. $P$-values are provided in brackets. *, **, and *** mark the $10 \%, 5 \%$, and $1 \%$ statistical significance for the estimated coefficients.

\begin{tabular}{|c|c|c|c|c|c|c|}
\hline \multirow[t]{2}{*}{ Recession measure } & \multicolumn{3}{|c|}{ Recession dummy } & \multicolumn{3}{|c|}{ Number of days in recession } \\
\hline & $\begin{array}{l}\text { Industry } \\
\text { fixed } \\
\text { effects }\end{array}$ & $\begin{array}{l}\text { Firm } \\
\text { fixed } \\
\text { effects }\end{array}$ & $\begin{array}{l}\text { Firm- } \\
\text { manager } \\
\text { fixed effects }\end{array}$ & $\begin{array}{l}\text { Industry } \\
\text { fixed } \\
\text { effects }\end{array}$ & $\begin{array}{l}\text { Firm } \\
\text { fixed } \\
\text { effects }\end{array}$ & $\begin{array}{l}\text { Firm- } \\
\text { manager } \\
\text { fixed effects }\end{array}$ \\
\hline $\log \left(\right.$ Delta $\left._{-1}\right)$ & $\begin{array}{c}0.007 \\
{[0.296]}\end{array}$ & $\begin{array}{l}0.017 \\
{[0.000]^{* * * *}}\end{array}$ & $\begin{array}{l}0.023 \\
{[0.000]^{* * * *}}\end{array}$ & $\begin{array}{c}0.006 \\
{[0.320]}\end{array}$ & $\begin{array}{l}0.017 \\
{[0.001]^{* * * *}}\end{array}$ & $\begin{array}{l}0.023 \\
{[0.000] * * *}\end{array}$ \\
\hline $\log \left(\operatorname{Vega}_{-1}\right)$ & $\begin{array}{l}-0.031 \\
{[0.000]^{* * *}}\end{array}$ & $\begin{array}{l}-0.047 \\
{[0.000] * * *}\end{array}$ & $\begin{array}{l}-0.046 \\
{[0.000]^{* * *}}\end{array}$ & $\begin{array}{l}-0.032 \\
{[0.000]^{* * *}}\end{array}$ & $\begin{array}{l}-0.047 \\
{[0.000] * * *}\end{array}$ & $\begin{array}{l}-0.046 \\
{[0.000]^{* * *}}\end{array}$ \\
\hline NBER recession measure & $\begin{array}{l}0.317 \\
{[0.000] * * *}\end{array}$ & $\begin{array}{l}0.332 \\
{[0.000]^{* * *}}\end{array}$ & $\begin{array}{l}0.283 \\
{[0.000]^{* * *}}\end{array}$ & $\begin{array}{l}0.064 \\
{[0.000]^{* * *}}\end{array}$ & $\begin{array}{l}0.066 \\
{[0.000] * * *}\end{array}$ & $\begin{array}{l}0.054 \\
{[0.000] * * *}\end{array}$ \\
\hline $\begin{array}{l}\log \left(\text { Delta }_{-1}\right) * \text { Recession } \\
\text { measure }\end{array}$ & $\begin{array}{l}-0.040 \\
{[0.000]^{* * *}}\end{array}$ & $\begin{array}{l}-0.027 \\
{[0.003] * * *}\end{array}$ & $\begin{array}{l}-0.023 \\
{[0.023]^{* *}}\end{array}$ & $\begin{array}{l}-0.007 \\
{[0.000] * *}\end{array}$ & $\begin{array}{l}-0.004 \\
{[0.006]^{* * *}}\end{array}$ & $\begin{array}{l}-0.003 \\
{[0.070]^{*}}\end{array}$ \\
\hline $\begin{array}{l}\log \left(\operatorname{Vega}_{-1}\right) * \text { Recession } \\
\text { measure }\end{array}$ & $\begin{array}{r}-0.002 \\
{[0.789]}\end{array}$ & $\begin{array}{l}-0.013 \\
{[0.084]^{*}}\end{array}$ & $\begin{array}{r}-0.003 \\
{[0.754]}\end{array}$ & $\begin{array}{r}-0.000 \\
{[0.972]}\end{array}$ & $\begin{array}{r}-0.002 \\
{[0.118]}\end{array}$ & $\begin{array}{r}-0.000 \\
{[0.817]}\end{array}$ \\
\hline $\begin{array}{l}\log (\text { Cash } \\
\left.\quad \text { compensation }_{-1}\right)\end{array}$ & $\begin{array}{l}-0.088 \\
{[0.000]^{* * *}}\end{array}$ & $\begin{array}{l}-0.072 \\
{[0.000] * * *}\end{array}$ & $\begin{array}{l}-0.058 \\
{[0.000]^{* * *}}\end{array}$ & $\begin{array}{l}-0.088 \\
{[0.000] * *}\end{array}$ & $\begin{array}{l}-0.072 \\
{[0.000] * * *}\end{array}$ & $\begin{array}{l}-0.058 \\
{[0.000]^{* * *}}\end{array}$ \\
\hline CEO tenure & $\begin{array}{c}-0.001 \\
{[0.502]}\end{array}$ & $\begin{array}{c}-0.002 \\
{[0.063]^{*}}\end{array}$ & $\begin{array}{c}0.014 \\
{[0.394]}\end{array}$ & $\begin{array}{c}-0.001 \\
{[0.495]}\end{array}$ & $\begin{array}{c}-0.002 \\
{[0.064]^{*}}\end{array}$ & $\begin{array}{c}0.014 \\
{[0.393]}\end{array}$ \\
\hline $\log ($ Sales $)$ & $\begin{array}{l}-0.172 \\
{[0.000]^{* * *}}\end{array}$ & $\begin{array}{l}-0.148 \\
{[0.000] * * *}\end{array}$ & $\begin{array}{l}-0.111 \\
{[0.000]^{* * *}}\end{array}$ & $\begin{array}{l}-0.172 \\
{[0.000] * * *}\end{array}$ & $\begin{array}{l}-0.148 \\
{[0.000]^{* * *}}\end{array}$ & $\begin{array}{l}-0.111 \\
{[0.000]^{* * *}}\end{array}$ \\
\hline Market-to-book & $\begin{array}{l}-0.012 \\
{[0.072]^{*}}\end{array}$ & $\begin{array}{c}0.008 \\
{[0.144]}\end{array}$ & $\begin{array}{c}-0.004 \\
{[0.540]}\end{array}$ & $\begin{array}{c}-0.012 \\
{[0.080]^{*}}\end{array}$ & $\begin{array}{c}0.009 \\
{[0.124]}\end{array}$ & $\begin{array}{c}-0.003 \\
{[0.592]}\end{array}$ \\
\hline R\&D expenditures/assets & $\begin{array}{l}1.864 \\
{[0.000]^{* * * *}}\end{array}$ & $\begin{array}{c}0.237 \\
{[0.076]^{*}}\end{array}$ & $\begin{array}{c}0.274 \\
{[0.067]^{*}}\end{array}$ & $\begin{array}{l}1.859 \\
{[0.000]^{* * *}}\end{array}$ & $\begin{array}{c}0.229 \\
{[0.088]^{*}}\end{array}$ & $\begin{array}{c}0.270 \\
{[0.073]^{*}}\end{array}$ \\
\hline Capital expenditures/assets & $\begin{array}{l}0.346 \\
{[0.029] * *}\end{array}$ & $\begin{array}{l}0.389 \\
{[0.004]^{* * * *}}\end{array}$ & $\begin{array}{l}0.404 \\
{[0.008]^{* * * *}}\end{array}$ & $\begin{array}{c}0.344 \\
{[0.030]^{* *}}\end{array}$ & $\begin{array}{l}0.384 \\
{[0.005]^{* * * *}}\end{array}$ & $\begin{array}{l}0.400 \\
{[0.009]^{* * *}}\end{array}$ \\
\hline Debt/assets & $\begin{array}{l}0.244 \\
{[0.000] * * *}\end{array}$ & $\begin{array}{l}0.296 \\
{[0.000]^{* * *}}\end{array}$ & $\begin{array}{l}0.397 \\
{[0.000]^{* * *}}\end{array}$ & $\begin{array}{l}0.246 \\
{[0.000]^{* * *}}\end{array}$ & $\begin{array}{l}0.299 \\
{[0.000]^{* * *}}\end{array}$ & $\begin{array}{l}0.399 \\
{[0.000]^{* * *}}\end{array}$ \\
\hline Constant & $\begin{array}{c}0.288 \\
{[0.152]}\end{array}$ & $\begin{array}{l}-0.722 \\
{[0.000] * * *}\end{array}$ & $\begin{array}{l}-1.036 \\
{[0.000]^{* * *}}\end{array}$ & $\begin{array}{l}0.555 \\
{[0.006] * * *}\end{array}$ & $\begin{array}{l}-0.723 \\
{[0.000] * * *}\end{array}$ & $\begin{array}{l}-1.033 \\
{[0.000]^{* * *}}\end{array}$ \\
\hline$N$ & 20,352 & 20,352 & 18,184 & 20,352 & 20,352 & 18,184 \\
\hline Year fixed effects & Yes & Yes & Yes & Yes & Yes & Yes \\
\hline$R$-squared & 0.550 & 0.515 & 0.534 & 0.550 & 0.515 & 0.534 \\
\hline Adjusted $R$-squared & 0.548 & 0.514 & 0.533 & 0.548 & 0.515 & 0.533 \\
\hline
\end{tabular}


Table IV. Relationship between managerial performance incentives, firm risk and the macroeconomic state

This table presents the results for the estimation of Equation (2) in the text. The dependent variable is firm risk, calculated as the logarithm of the annualized variance of the daily stock returns. The main variable of interest is the interaction of logarithm of the lagged value of delta with the macroeconomic state measure. The definitions of the variables are provided in Appendix A. All regressions control for year fixed effects. Robust standard errors are clustered at firm level in regressions that control for industry fixed effects and at firm-manager level in regressions that control for firm-manager pair fixed effects. $P$-values are provided in brackets. ${ }^{*}, * *$, and $*^{* *}$ mark the $10 \%, 5 \%$, and $1 \%$ statistical significance for the estimated coefficients.

\begin{tabular}{|c|c|c|c|c|c|c|}
\hline \multirow{2}{*}{$\begin{array}{l}\text { Macroeconomic } \\
\text { state measured by }\end{array}$} & \multicolumn{3}{|l|}{ GDP growth } & \multicolumn{3}{|c|}{ Non-farm payroll } \\
\hline & $\begin{array}{l}\text { Industry } \\
\text { fixed } \\
\text { effects }\end{array}$ & $\begin{array}{l}\text { Firm } \\
\text { fixed } \\
\text { effects }\end{array}$ & $\begin{array}{l}\text { Firm- } \\
\text { manager } \\
\text { fixed effects }\end{array}$ & $\begin{array}{l}\text { Industry } \\
\text { fixed } \\
\text { effects }\end{array}$ & $\begin{array}{l}\text { Firm } \\
\text { fixed } \\
\text { effects }\end{array}$ & $\begin{array}{l}\text { Firm- } \\
\text { manager } \\
\text { fixed effects }\end{array}$ \\
\hline $\log \left(\right.$ Delta $\left._{-1}\right)$ & $\begin{array}{l}-0.013 \\
{[0.033]^{* *}}\end{array}$ & $\begin{array}{c}0.005 \\
{[0.365]}\end{array}$ & $\begin{array}{c}0.012 \\
{[0.063]^{*}}\end{array}$ & $\begin{array}{l}0.016 \\
{[0.016]^{* *}}\end{array}$ & $\begin{array}{l}0.025 \\
{[0.000]^{* * *}}\end{array}$ & $\begin{array}{l}0.030 \\
{[0.000] * * *}\end{array}$ \\
\hline $\log \left(\operatorname{Vega}_{-1}\right)$ & $\begin{array}{l}-0.031 \\
{[0.000]^{* * *}}\end{array}$ & $\begin{array}{l}-0.051 \\
{[0.000]^{* * *}}\end{array}$ & $\begin{array}{l}-0.046 \\
{[0.000]^{* * *}}\end{array}$ & $\begin{array}{l}-0.032 \\
{[0.000]^{* * *}}\end{array}$ & $\begin{array}{l}-0.047 \\
{[0.000]^{* * *}}\end{array}$ & $\begin{array}{l}-0.049 \\
{[0.000]^{* * *}}\end{array}$ \\
\hline Macroeconomy & $\begin{array}{l}-0.123 \\
{[0.000]^{* * *}}\end{array}$ & $\begin{array}{l}-0.117 \\
{[0.000] * * *}\end{array}$ & $\begin{array}{l}-0.111 \\
{[0.000]^{* * *}}\end{array}$ & $\begin{array}{l}-0.001 \\
{[0.000]^{*} * *}\end{array}$ & $\begin{array}{l}-0.001 \\
{[0.000]^{* * *}}\end{array}$ & $\begin{array}{l}-0.001 \\
{[0.000]^{* * *}}\end{array}$ \\
\hline $\begin{array}{l}\log \left(\text { Delta }_{-1}\right) * \\
\quad \text { Macroeconomic state }\end{array}$ & $\begin{array}{c}0.014 \\
{[0.000]^{* * *}}\end{array}$ & $\begin{array}{l}0.009 \\
{[0.000]^{* * *}}\end{array}$ & $\begin{array}{l}0.009 \\
{[0.000]^{* * * *}}\end{array}$ & $\begin{array}{l}0.112 \\
{[0.000]^{* * *}}\end{array}$ & $\begin{array}{l}0.075 \\
{[0.000]^{* * *}}\end{array}$ & $\begin{array}{l}0.063 \\
{[0.002]^{* * *}}\end{array}$ \\
\hline $\begin{array}{l}\log \left(\operatorname{Vega}_{-1}\right) * \\
\quad \text { Macroeconomic state }\end{array}$ & $\begin{array}{r}-0.000 \\
{[0.813]}\end{array}$ & $\begin{array}{c}0.002 \\
{[0.313]}\end{array}$ & $\begin{array}{c}-0.000 \\
{[0.880]}\end{array}$ & $\begin{array}{r}-0.008 \\
{[0.614]}\end{array}$ & $\begin{array}{c}0.011 \\
{[0.467]}\end{array}$ & $\begin{array}{r}-0.013 \\
{[0.439]}\end{array}$ \\
\hline $\log \left(\right.$ Cash compensation $\left._{-1}\right)$ & $\begin{array}{l}-0.091 \\
{[0.000]^{* * *}}\end{array}$ & $\begin{array}{l}-0.075 \\
{[0.000]^{* * *}}\end{array}$ & $\begin{array}{l}-0.061 \\
{[0.000]^{* * *}}\end{array}$ & $\begin{array}{l}-0.090 \\
{[0.000] * * *}\end{array}$ & $\begin{array}{l}-0.075 \\
{[0.000] * * *}\end{array}$ & $\begin{array}{l}-0.061 \\
{[0.000]^{* * *}}\end{array}$ \\
\hline CEO tenure & $\begin{array}{r}-0.001 \\
{[0.516]}\end{array}$ & $\begin{array}{c}-0.002 \\
{[0.067]^{*}}\end{array}$ & $\begin{array}{c}0.012 \\
{[0.446]}\end{array}$ & $\begin{array}{r}-0.001 \\
{[0.506]}\end{array}$ & $\begin{array}{l}-0.002 \\
{[0.055]^{*}}\end{array}$ & $\begin{array}{c}0.012 \\
{[0.432]}\end{array}$ \\
\hline $\log ($ Sales $)$ & $\begin{array}{l}-0.171 \\
{[0.000]^{* * *}}\end{array}$ & $\begin{array}{l}-0.143 \\
{[0.000] * * *}\end{array}$ & $\begin{array}{l}-0.106 \\
{[0.000]^{* * *}}\end{array}$ & $\begin{array}{l}-0.172 \\
{[0.000]^{*} * *}\end{array}$ & $\begin{array}{l}-0.145 \\
{[0.000]^{* * *}}\end{array}$ & $\begin{array}{l}-0.109 \\
{[0.000] * * *}\end{array}$ \\
\hline Market-to-book & $\begin{array}{l}-0.013 \\
{[0.056]^{*}}\end{array}$ & $\begin{array}{c}0.008 \\
{[0.150]}\end{array}$ & $\begin{array}{c}-0.004 \\
{[0.543]}\end{array}$ & $\begin{array}{c}-0.012 \\
{[0.071]^{*}}\end{array}$ & $\begin{array}{c}0.009 \\
{[0.097]^{*}}\end{array}$ & $\begin{array}{c}-0.002 \\
{[0.702]}\end{array}$ \\
\hline R\&D expenditures/assets & $\begin{array}{l}1.861 \\
{[0.000]^{* * *}}\end{array}$ & $\begin{array}{c}0.233 \\
{[0.074]^{*}}\end{array}$ & $\begin{array}{c}0.277 \\
{[0.063]^{*}}\end{array}$ & $\begin{array}{l}1.853 \\
{[0.000]^{* * *}}\end{array}$ & $\begin{array}{c}0.217 \\
{[0.096]^{*}}\end{array}$ & $\begin{array}{c}0.261 \\
{[0.077]^{*}}\end{array}$ \\
\hline Capital expenditures/assets & $\begin{array}{c}0.348 \\
{[0.028]^{* *}}\end{array}$ & $\begin{array}{c}0.381 \\
{[0.005]^{* * *}}\end{array}$ & $\begin{array}{c}0.388 \\
{[0.012]^{* *}}\end{array}$ & $\begin{array}{l}0.327 \\
{[0.039]^{* *}}\end{array}$ & $\begin{array}{l}0.351 \\
{[0.010]^{* * *}}\end{array}$ & $\begin{array}{l}0.360 \\
{[0.018]^{* *}}\end{array}$ \\
\hline Leverage ratio & $\begin{array}{l}0.243 \\
{[0.000]^{* * *}}\end{array}$ & $\begin{array}{l}0.298 \\
{[0.000]^{* * *}}\end{array}$ & $\begin{array}{l}0.396 \\
{[0.000]^{* * *}}\end{array}$ & $\begin{array}{l}0.242 \\
{[0.000] * * *}\end{array}$ & $\begin{array}{l}0.300 \\
{[0.000]^{* * *}}\end{array}$ & $\begin{array}{l}0.399 \\
{[0.000]^{* * * *}}\end{array}$ \\
\hline Constant & $\begin{array}{c}0.236 \\
{[0.241]}\end{array}$ & $\begin{array}{l}-0.556 \\
{[0.000]^{* * *}}\end{array}$ & $\begin{array}{l}-0.873 \\
{[0.000]^{* * *}}\end{array}$ & $\begin{array}{l}-0.573 \\
{[0.004]^{* * *}}\end{array}$ & $\begin{array}{l}-0.793 \\
{[0.000]^{* * *}}\end{array}$ & $\begin{array}{l}-1.089 \\
{[0.000]^{* * *}}\end{array}$ \\
\hline$N$ & 20,352 & 20,352 & 18,184 & 20,352 & 20,352 & 18,184 \\
\hline Year fixed effects & Yes & Yes & Yes & Yes & Yes & Yes \\
\hline$R$-squared & 0.554 & 0.520 & 0.539 & 0.554 & 0.522 & 0.541 \\
\hline Adjusted $R$-squared & 0.552 & 0.519 & 0.539 & 0.552 & 0.521 & 0.541 \\
\hline
\end{tabular}


Table IV. Continued

\begin{tabular}{|c|c|c|c|c|c|c|}
\hline \multirow{2}{*}{$\begin{array}{l}\text { Macroeconomic } \\
\text { state measured by: }\end{array}$} & \multicolumn{3}{|c|}{ Personal expenditure growth } & \multicolumn{3}{|c|}{ Retail sales growth } \\
\hline & $\begin{array}{l}\text { Industry } \\
\text { fixed } \\
\text { effects }\end{array}$ & $\begin{array}{l}\text { Firm } \\
\text { fixed } \\
\text { effects }\end{array}$ & $\begin{array}{l}\text { Firm- } \\
\text { manager } \\
\text { fixed effects }\end{array}$ & $\begin{array}{l}\text { Industry } \\
\text { fixed } \\
\text { effects }\end{array}$ & $\begin{array}{l}\text { Firm } \\
\text { fixed } \\
\text { effects }\end{array}$ & $\begin{array}{l}\text { Firm- } \\
\text { manager } \\
\text { fixed effects }\end{array}$ \\
\hline $\log \left(\right.$ Delta $\left._{-1}\right)$ & $\begin{array}{c}0.011 \\
{[0.085]^{*}}\end{array}$ & $\begin{array}{l}0.020 \\
{[0.000]^{* * * *}}\end{array}$ & $\begin{array}{l}0.026 \\
{[0.000] * * *}\end{array}$ & $\begin{array}{l}0.025 \\
{[0.001]^{* * *}}\end{array}$ & $\begin{array}{l}0.030 \\
{[0.000]^{* * *}}\end{array}$ & $\begin{array}{l}0.033 \\
{[0.000] * * *}\end{array}$ \\
\hline $\log \left(\operatorname{Vega}_{-1}\right)$ & $\begin{array}{l}-0.031 \\
{[0.000] * *}\end{array}$ & $\begin{array}{l}-0.049 \\
{[0.000]^{* * *}}\end{array}$ & $\begin{array}{l}-0.047 \\
{[0.000] * *}\end{array}$ & $\begin{array}{l}-0.027 \\
{[0.001]^{* * *}}\end{array}$ & $\begin{array}{l}-0.042 \\
{[0.000] * * *}\end{array}$ & $\begin{array}{l}-0.044 \\
{[0.000] * *}\end{array}$ \\
\hline Macroeconomy & $\begin{array}{l}-0.276 \\
{[0.000]^{* * *}}\end{array}$ & $\begin{array}{l}-0.223 \\
{[0.000]^{* * *}}\end{array}$ & $\begin{array}{l}-0.184 \\
{[0.000]^{* * *}}\end{array}$ & $\begin{array}{l}-0.183 \\
{[0.000] * * *}\end{array}$ & $\begin{array}{l}-0.154 \\
{[0.000] * * *}\end{array}$ & $\begin{array}{l}-0.121 \\
{[0.000]^{* * *}}\end{array}$ \\
\hline $\begin{array}{l}\log \left(\text { Delta }_{-1}\right) * \\
\quad \text { Macroeconomic state }\end{array}$ & $\begin{array}{l}0.036 \\
{[0.000]^{* * *}}\end{array}$ & $\begin{array}{l}0.023 \\
{[0.000]^{* * *}}\end{array}$ & $\begin{array}{l}0.019 \\
{[0.001]^{* * *}}\end{array}$ & $\begin{array}{l}0.022 \\
{[0.000]^{* * *}}\end{array}$ & $\begin{array}{l}0.015 \\
{[0.000]^{* * *}}\end{array}$ & $\begin{array}{l}0.012 \\
{[0.001]^{* * * *}}\end{array}$ \\
\hline $\begin{array}{l}\log \left(\operatorname{Vega}_{-1}\right) * \\
\quad \text { Macroeconomic state }\end{array}$ & $\begin{array}{c}0.000 \\
{[1.000]}\end{array}$ & $\begin{array}{c}0.004 \\
{[0.379]}\end{array}$ & $\begin{array}{r}-0.000 \\
{[0.923]}\end{array}$ & $\begin{array}{c}0.003 \\
{[0.349]}\end{array}$ & $\begin{array}{l}0.006 \\
{[0.040]^{* *}}\end{array}$ & $\begin{array}{c}0.002 \\
{[0.574]}\end{array}$ \\
\hline $\log _{(\text {Cash compensation }}$ co $\left._{1}\right)$ & $\begin{array}{l}-0.089 \\
{[0.000]^{* * *}}\end{array}$ & $\begin{array}{l}-0.073 \\
{[0.000]^{* * *}}\end{array}$ & $\begin{array}{l}-0.058 \\
{[0.000]^{*} *}\end{array}$ & $\begin{array}{l}-0.090 \\
{[0.000]^{* * *}}\end{array}$ & $\begin{array}{l}-0.072 \\
{[0.000] * * *}\end{array}$ & $\begin{array}{l}-0.058 \\
{[0.000]^{* * *}}\end{array}$ \\
\hline CEO tenure & $\begin{array}{r}-0.001 \\
{[0.516]}\end{array}$ & $\begin{array}{l}-0.002 \\
{[0.055]^{*}}\end{array}$ & $\begin{array}{c}0.014 \\
{[0.377]}\end{array}$ & $\begin{array}{c}-0.001 \\
{[0.559]}\end{array}$ & $\begin{array}{l}-0.002 \\
{[0.061]^{*}}\end{array}$ & $\begin{array}{c}0.014 \\
{[0.389]}\end{array}$ \\
\hline $\log ($ Sales $)$ & $\begin{array}{l}-0.172 \\
{[0.000] * *}\end{array}$ & $\begin{array}{l}-0.148 \\
{[0.000]^{* * *}}\end{array}$ & $\begin{array}{l}-0.111 \\
{[0.000] * * *}\end{array}$ & $\begin{array}{l}-0.172 \\
{[0.000] * * *}\end{array}$ & $\begin{array}{l}-0.147 \\
{[0.000] * * *}\end{array}$ & $\begin{array}{l}-0.111 \\
{[0.000]^{* * *}}\end{array}$ \\
\hline Market-to-book & $\begin{array}{l}-0.012 \\
{[0.071]^{*}}\end{array}$ & $\begin{array}{c}0.008 \\
{[0.154]}\end{array}$ & $\begin{array}{c}-0.004 \\
{[0.533]}\end{array}$ & $\begin{array}{c}-0.012 \\
{[0.079]^{*}}\end{array}$ & $\begin{array}{c}0.008 \\
{[0.161]}\end{array}$ & $\begin{array}{c}-0.004 \\
{[0.521]}\end{array}$ \\
\hline $\mathrm{R} \& \mathrm{D}$ expenditures/assets & $\begin{array}{l}1.865 \\
{[0.000]^{* * *}}\end{array}$ & $\begin{array}{c}0.235 \\
{[0.078]^{*}}\end{array}$ & $\begin{array}{c}0.274 \\
{[0.066]^{*}}\end{array}$ & $\begin{array}{l}1.868 \\
{[0.000]^{* * *}}\end{array}$ & $\begin{array}{c}0.238 \\
{[0.071]^{*}}\end{array}$ & $\begin{array}{c}0.278 \\
{[0.061]^{*}}\end{array}$ \\
\hline Capital expenditures/assets & $\begin{array}{c}0.338 \\
{[0.034]^{* *}}\end{array}$ & $\begin{array}{l}0.371 \\
{[0.007]^{* * * *}}\end{array}$ & $\begin{array}{c}0.386 \\
{[0.012]^{* *}}\end{array}$ & $\begin{array}{l}0.342 \\
{[0.031]^{* *}}\end{array}$ & $\begin{array}{l}0.372 \\
{[0.006]^{* * * *}}\end{array}$ & $\begin{array}{l}0.389 \\
{[0.011]^{* *}}\end{array}$ \\
\hline Leverage ratio & $\begin{array}{l}0.244 \\
{[0.000]^{* * *}}\end{array}$ & $\begin{array}{l}0.298 \\
{[0.000]^{* * *}}\end{array}$ & $\begin{array}{l}0.399 \\
{[0.000]^{* * *}}\end{array}$ & $\begin{array}{l}0.242 \\
{[0.000]^{* * *}}\end{array}$ & $\begin{array}{l}0.296 \\
{[0.000]^{* * *}}\end{array}$ & $\begin{array}{l}0.399 \\
{[0.000]^{* * *}}\end{array}$ \\
\hline Constant & $\begin{array}{l}0.648 \\
{[0.001]^{* * *}}\end{array}$ & $\begin{array}{l}-0.742 \\
{[0.000]^{* * *}}\end{array}$ & $\begin{array}{l}-1.062 \\
{[0.000] * * *}\end{array}$ & $\begin{array}{c}0.176 \\
{[0.369]}\end{array}$ & $\begin{array}{l}-0.867 \\
{[0.000] * * *}\end{array}$ & $\begin{array}{l}-1.147 \\
{[0.000] * *}\end{array}$ \\
\hline$N$ & 20,352 & 20,352 & 18,184 & 20,352 & 20,352 & 18,184 \\
\hline Year fixed effects & Yes & Yes & Yes & Yes & Yes & Yes \\
\hline$R$-squared & 0.551 & 0.516 & 0.535 & 0.552 & 0.517 & 0.535 \\
\hline Adjusted $R$-squared & 0.549 & 0.515 & 0.534 & 0.550 & 0.516 & 0.535 \\
\hline
\end{tabular}

In Table V, we summarize the results. Similar to the earlier results, we obtain a negative and statistically significant coefficient for the interaction terms when use the NBER recession dates (Columns 1 and 2). For example, the coefficient on the interaction term for the NBER dummy is -0.041 and indicates that a $1 \%$ increase in delta is associated with a $0.041 \%$ reduction in firm-specific risk during recessions. Accordingly, an increase in delta from its 25 th percentile to its 75 th percentile would be associated with a $10 \%$ increase in firm risk during expansion years but with a $20 \%$ decrease in risk during recession years. We present the findings using the GDP growth rate, additions to non-farm payroll, and personal consumption expenditure growth rate as a proxy for the macroeconomic state in 
Table V. Relationship between managerial performance incentives, firm-specific risk, and the macroeconomic state

This table presents the results for the estimation of Equation (3) in the text. The dependent variable is firm-specific (idiosyncatic) risk, calculated as the logarithm of the annualized variance of residuals from the market model. The main variable of interest is the interaction of logarithm of the lagged value of delta with the macroeconomic state measure. The definitions of the variables are provided in Appendix A. All regressions control for year fixed effects. Robust standard errors are clustered at the firm-manager level. $P$-values are provided in brackets. ${ }^{*}{ }^{* *}$, and ${ }^{* * *}$ mark the $10 \%, 5 \%$, and $1 \%$ statistical significance for the estimated coefficients.

\begin{tabular}{|c|c|c|c|c|c|c|}
\hline \multirow[b]{2}{*}{$\begin{array}{l}\text { Macroeconomic } \\
\text { state measured by: }\end{array}$} & \multicolumn{6}{|c|}{ Idiosyncratic risk } \\
\hline & $\begin{array}{l}\text { NBER } \\
\text { recession } \\
\text { dummy }\end{array}$ & $\begin{array}{l}\text { NBER } \\
\text { recession } \\
\text { days }\end{array}$ & $\begin{array}{l}\text { GDP } \\
\text { growth }\end{array}$ & $\begin{array}{l}\text { Non-farm } \\
\text { payroll }\end{array}$ & $\begin{array}{l}\text { Personal } \\
\text { expenditure } \\
\text { growth }\end{array}$ & $\begin{array}{l}\text { Retail } \\
\text { sales } \\
\text { growth }\end{array}$ \\
\hline $\log \left(\right.$ Delta $\left._{-1}\right)$ & $\begin{array}{l}0.014 \\
{[0.016]^{* *}}\end{array}$ & $\begin{array}{l}0.013 \\
{[0.021]^{* *}}\end{array}$ & $\begin{array}{r}-0.004 \\
{[0.544]}\end{array}$ & $\begin{array}{l}0.023 \\
{[0.000] * * *}\end{array}$ & $\begin{array}{l}0.016 \\
{[0.005]^{* * *}}\end{array}$ & $\begin{array}{l}0.027 \\
{[0.000] * * *}\end{array}$ \\
\hline $\log \left(\operatorname{Vega}_{-1}\right)$ & $\begin{array}{l}-0.044 \\
{[0.000] * * *}\end{array}$ & $\begin{array}{l}-0.044 \\
{[0.000] * * *}\end{array}$ & $\begin{array}{l}-0.045 \\
{[0.000]^{* * *}}\end{array}$ & $\begin{array}{l}-0.044 \\
{[0.000] * * *}\end{array}$ & $\begin{array}{l}-0.044 \\
{[0.000] * * *}\end{array}$ & $\begin{array}{l}-0.042 \\
{[0.000]^{* * *}}\end{array}$ \\
\hline Macroeconomy & $\begin{array}{l}0.369 \\
{[0.000]^{* * *}}\end{array}$ & $\begin{array}{l}0.069 \\
{[0.000]^{* * *}}\end{array}$ & $\begin{array}{l}-0.113 \\
{[0.000]^{* * *}}\end{array}$ & $\begin{array}{l}-0.001 \\
{[0.000]^{* * *}}\end{array}$ & $\begin{array}{l}-0.198 \\
{[0.000]^{* * *}}\end{array}$ & $\begin{array}{l}-0.131 \\
{[0.000] * * *}\end{array}$ \\
\hline $\begin{array}{l}\log \left(\text { Delta }_{-1}\right) * \\
\quad \text { Macroeconomic state }\end{array}$ & $\begin{array}{l}-0.041 \\
{[0.000] * * *}\end{array}$ & $\begin{array}{l}-0.007 \\
{[0.001]^{* * *}}\end{array}$ & $\begin{array}{l}0.012 \\
{[0.000]^{* * * *}}\end{array}$ & $\begin{array}{l}0.102 \\
{[0.000] * * *}\end{array}$ & $\begin{array}{l}0.027 \\
{[0.000]^{* * *}}\end{array}$ & $\begin{array}{l}0.017 \\
{[0.000]^{* * *}}\end{array}$ \\
\hline $\begin{array}{l}\log \left(\operatorname{Vega}_{-1}\right) * \\
\quad \text { Macroeconomic state }\end{array}$ & $\begin{array}{r}-0.004 \\
{[0.616]}\end{array}$ & $\begin{array}{r}-0.001 \\
{[0.563]}\end{array}$ & $\begin{array}{c}0.001 \\
{[0.723]}\end{array}$ & $\begin{array}{r}-0.002 \\
{[0.913]}\end{array}$ & $\begin{array}{c}0.001 \\
{[0.827]}\end{array}$ & $\begin{array}{c}0.002 \\
{[0.520]}\end{array}$ \\
\hline $\log _{(\text {Cash compensation }}$ co $_{-1}$ & $\begin{array}{l}-0.069 \\
{[0.000] * * *}\end{array}$ & $\begin{array}{l}-0.069 \\
{[0.000]^{* * *}}\end{array}$ & $\begin{array}{l}-0.073 \\
{[0.000]^{* * *}}\end{array}$ & $\begin{array}{l}-0.073 \\
{[0.000] * *}\end{array}$ & $\begin{array}{l}-0.069 \\
{[0.000] * * *}\end{array}$ & $\begin{array}{l}-0.069 \\
{[0.000]^{* * *}}\end{array}$ \\
\hline CEO tenure & $\begin{array}{c}0.016 \\
{[0.285]}\end{array}$ & $\begin{array}{c}0.016 \\
{[0.283]}\end{array}$ & $\begin{array}{c}0.015 \\
{[0.327]}\end{array}$ & $\begin{array}{c}0.015 \\
{[0.312]}\end{array}$ & $\begin{array}{c}0.017 \\
{[0.259]}\end{array}$ & $\begin{array}{c}0.016 \\
{[0.273]}\end{array}$ \\
\hline $\log ($ Sales $)$ & $\begin{array}{l}-0.125 \\
{[0.000] * *}\end{array}$ & $\begin{array}{l}-0.125 \\
{[0.000]^{* * *}}\end{array}$ & $\begin{array}{l}-0.121 \\
{[0.000]^{* * *}}\end{array}$ & $\begin{array}{l}-0.123 \\
{[0.000]^{* * *}}\end{array}$ & $\begin{array}{l}-0.126 \\
{[0.000]^{* * *}}\end{array}$ & $\begin{array}{l}-0.125 \\
{[0.000]^{* * *}}\end{array}$ \\
\hline Market-to-book & $\begin{array}{l}-0.016 \\
{[0.009] * * *}\end{array}$ & $\begin{array}{l}-0.015 \\
{[0.011]^{*} *}\end{array}$ & $\begin{array}{l}-0.017 \\
{[0.006]^{* * *}}\end{array}$ & $\begin{array}{l}-0.015 \\
{[0.011]^{* *}}\end{array}$ & $\begin{array}{l}-0.016 \\
{[0.007]^{* * *}}\end{array}$ & $\begin{array}{l}-0.016 \\
{[0.007]^{* * *}}\end{array}$ \\
\hline R\&D expenditures/assets & $\begin{array}{l}0.348 \\
{[0.024]^{* *}}\end{array}$ & $\begin{array}{l}0.343 \\
{[0.027]^{* *}}\end{array}$ & $\begin{array}{l}0.353 \\
{[0.020]^{* * *}}\end{array}$ & $\begin{array}{l}0.336 \\
{[0.026]^{* * *}}\end{array}$ & $\begin{array}{l}0.346 \\
{[0.024]^{* * *}}\end{array}$ & $\begin{array}{l}0.353 \\
{[0.020]^{* *}}\end{array}$ \\
\hline Capital expenditures/assets & $\begin{array}{c}0.334 \\
{[0.032]^{* *}}\end{array}$ & $\begin{array}{l}0.330 \\
{[0.034]^{* *}}\end{array}$ & $\begin{array}{l}0.319 \\
{[0.041]^{* *}}\end{array}$ & $\begin{array}{c}0.302 \\
{[0.051]^{*}}\end{array}$ & $\begin{array}{c}0.320 \\
{[0.039]^{* *}}\end{array}$ & $\begin{array}{l}0.322 \\
{[0.038]^{* *}}\end{array}$ \\
\hline Leverage ratio & $\begin{array}{l}0.431 \\
{[0.000]^{* * *}}\end{array}$ & $\begin{array}{l}0.434 \\
{[0.000] * * *}\end{array}$ & $\begin{array}{l}0.433 \\
{[0.000]^{* * * *}}\end{array}$ & $\begin{array}{l}0.435 \\
{[0.000] * * *}\end{array}$ & $\begin{array}{l}0.435 \\
{[0.000]^{* * *}}\end{array}$ & $\begin{array}{l}0.434 \\
{[0.000] * * *}\end{array}$ \\
\hline Constant & $\begin{array}{l}-0.882 \\
{[0.000] * *}\end{array}$ & $\begin{array}{l}-0.880 \\
{[0.000]^{* * *}}\end{array}$ & $\begin{array}{l}-0.702 \\
{[0.000]^{* * *}}\end{array}$ & $\begin{array}{l}-0.938 \\
{[0.000] * *}\end{array}$ & $\begin{array}{l}-0.895 \\
{[0.000] * *}\end{array}$ & $\begin{array}{l}-0.990 \\
{[0.000]^{* * *}}\end{array}$ \\
\hline$N$ & 18,184 & 18,184 & 18,184 & 18,184 & 18,184 & 18,184 \\
\hline Year fixed effects & Yes & Yes & Yes & Yes & Yes & Yes \\
\hline Firm-manager fixed effects & Yes & Yes & Yes & Yes & Yes & Yes \\
\hline$R$-squared & 0.495 & 0.495 & 0.500 & 0.500 & 0.495 & 0.496 \\
\hline Adjusted $R$-squared & 0.494 & 0.494 & 0.499 & 0.499 & 0.494 & 0.495 \\
\hline
\end{tabular}


Columns 3, 4, and 5, respectively. We obtain a positive coefficient on the interactions terms across all specifications and the estimates are significant at the $1 \%$ level.

\subsection{Endogeneity}

We have two endogeneity concerns specific to this study. First, the relationship between firm risk and pay-for-performance sensitivity might be correlated with a factor related to economic growth, and consequently the interaction of delta and the macroeconomic state may be endogenous with respect to risk taking. Second, the macroeconomic state may have a direct effect on the value of managerial incentives because during recessions lower stock prices would lead to a mechanical decrease in performance incentives (delta) for a fixed amount of stock and stock option holdings. Since, on average, firm risk increases in recessions, the weakening of the delta-risk relationship that we uncover may simply be a consequence of the increase in the value of our dependent variable accompanying the mechanical decline in the value of delta during recessions. Due to these concerns, we account for the endogenous nature of the incentive contracts using estimation methods specifically designed to deal with endogeneity-namely instrumental variables and simultaneous equations estimations.

\section{2.a. Instrumental variables regressions}

We treat CEO incentives as endogenous and estimate the contract design and risk equations using two-stage least squares (2SLS). Our choices of instruments for delta and vega are based on the determinants identified by the previous literature and include surplus cash (Core and Guay, 1999; Coles, Daniel, and Naveen, 2006; Armstrong and Vashishtha, 2012), tax-loss carry forwards (Armstrong and Vashishtha, 2012), and the accounting cost that public firms incurred by the implementation of FAS 123R (Hayes, Lemmon, and Qiu, 2012). We expect these variables only to have an indirect relationship with firm risk through their effect on equity incentives and the other control variables. We confirm the econometric validity of these instruments by conducting Hansen's tests of overidentifying restrictions (Hansen, 1982). We present the 2SLS results in Table VI.

Surplus cash is a proxy for cash constraints of a firm, which can influence the CEO's equity incentives since cash-constrained firms tend to substitute cash compensation with restricted stock and stock options (Core and Guay, 1999). However, the presence (or lack) of cash constraints by itself does not necessarily imply systematically different levels of firm risk. Still, one may argue that cash surplus may not be a valid instrument if risky firms keep a larger cash cushion to avoid financial distress and at the same time provide more equity incentives to their CEOs in order to deal with the agency problems associated with free cash flow. However, Armstrong and Vashishtha (2012) present evidence that the results of the 2SLS regressions are unchanged after removing the variation in cash balances due to precautionary motives by regressing firms' cash balance on an index of financial constraints.

The tax-loss carry forward indicator is a proxy for the firm's marginal tax rate. Firms with lower tax rates have more incentive to provide equity-based compensation. This is because Section 162(m) of the US Internal Revenue Code limits the annual tax deduction to $\$ 1$ million for compensation paid to a public company's CEO and the three highest compensated officers (other than the CFO) but excludes the grants of options and restricted stock (i.e., sources of delta). We do not expect tax-loss carry forward to have a direct effect on firm risk. 
Table VI. Endogeneity

This table presents the results for the estimation of Equation (2) and Equation (3) in the text by two-stage and three-stage least squares. In Columns 1 and 3 , the dependent variable is firm risk, calculated as the logarithm of the annualized variance of the daily stock returns. In Columns 2 and 4, the dependent variable is firm-specific (idiosyncatic) risk, calculated as the logarithm of the annualized variance of residuals from the market model. The main variable of interest is the interaction of logarithm of the lagged value of delta with the macroeconomic state measure. Macroeconomic state is measured as the real GDP growth rate. The definitions of the variables are provided in Appendix A. All regressions control for year fixed effects. Robust standard errors are clustered at firm level in regressions that control for industry fixed effects and at firm-manager level in regressions that control for firm-manager pair fixed effects. $P$-values are provided in brackets. ${ }^{*},{ }^{*}$, and ${ }^{* * *}$ mark the $10 \%, 5 \%$, and $1 \%$ statistical significance for the estimated coefficients.

\begin{tabular}{|c|c|c|c|c|}
\hline \multirow[t]{2}{*}{ Estimation method } & \multicolumn{2}{|c|}{ Two-stage least squares } & \multicolumn{2}{|c|}{ Three-stage least squares } \\
\hline & Total risk & $\begin{array}{l}\text { Firm-specific } \\
\text { risk }\end{array}$ & Total risk & $\begin{array}{l}\text { Firm-specific } \\
\text { risk }\end{array}$ \\
\hline $\log ($ Delta $)$ & $\begin{array}{c}1.198 \\
{[0.113]}\end{array}$ & $\begin{array}{c}1.077 \\
{[0.123]}\end{array}$ & $\begin{array}{c}0.069 \\
{[0.087]^{*}}\end{array}$ & $\begin{array}{l}-0.102 \\
{[0.018]^{* *}}\end{array}$ \\
\hline $\log ($ Vega $)$ & $\begin{array}{c}-1.266 \\
{[0.072]^{*}}\end{array}$ & $\begin{array}{c}-1.168 \\
{[0.072]^{*}}\end{array}$ & $\begin{array}{l}-0.214 \\
{[0.000]^{* * *}}\end{array}$ & $\begin{array}{l}-0.097 \\
{[0.000]^{* * *}}\end{array}$ \\
\hline Macroeconomy & $\begin{array}{l}-0.444 \\
{[0.000]^{* * *}}\end{array}$ & $\begin{array}{l}-0.425 \\
{[0.000]^{* * *}}\end{array}$ & $\begin{array}{l}-0.109 \\
{[0.000]^{* * *}}\end{array}$ & $\begin{array}{l}-0.229 \\
{[0.000]^{* * *}}\end{array}$ \\
\hline Log(Delta) * Macroeconomic state & $\begin{array}{l}0.099 \\
{[0.010]^{* * *}}\end{array}$ & $\begin{array}{c}0.094 \\
{[0.008]^{* * *}}\end{array}$ & $\begin{array}{c}0.021 \\
{[0.001]^{* * *}}\end{array}$ & $\begin{array}{c}0.053 \\
{[0.000]^{* * *}}\end{array}$ \\
\hline $\log ($ Vega $) *$ Macroeconomic state & $\begin{array}{c}-0.042 \\
{[0.329]}\end{array}$ & $\begin{array}{c}-0.033 \\
{[0.401]}\end{array}$ & $\begin{array}{c}-0.003 \\
{[0.353]}\end{array}$ & $\begin{array}{l}-0.010 \\
{[0.005]^{* * *}}\end{array}$ \\
\hline Log(Cash compensation) & $\begin{array}{l}-0.326 \\
{[0.020]^{* *}}\end{array}$ & $\begin{array}{l}-0.313 \\
{[0.017]^{* *}}\end{array}$ & $\begin{array}{c}-0.013 \\
{[0.409]}\end{array}$ & $\begin{array}{l}-0.066 \\
{[0.000]^{* * *}}\end{array}$ \\
\hline CEO tenure & $\begin{array}{c}0.068 \\
{[0.313]}\end{array}$ & $\begin{array}{c}0.065 \\
{[0.293]}\end{array}$ & $\begin{array}{l}-0.010 \\
{[0.000]^{* * *}}\end{array}$ & $\begin{array}{c}0.001 \\
{[0.792]}\end{array}$ \\
\hline $\log ($ Sales $)$ & $\begin{array}{c}-0.150 \\
{[0.190]}\end{array}$ & $\begin{array}{c}-0.157 \\
{[0.162]}\end{array}$ & $\begin{array}{l}-0.144 \\
{[0.000]^{* * *}}\end{array}$ & $\begin{array}{l}-0.157 \\
{[0.000]^{* * *}}\end{array}$ \\
\hline Market-to-book & $\begin{array}{c}-0.342 \\
{[0.099]^{*}}\end{array}$ & $\begin{array}{c}-0.321 \\
{[0.094]^{*}}\end{array}$ & $\begin{array}{l}-0.024 \\
{[0.042]^{* *}}\end{array}$ & $\begin{array}{c}0.001 \\
{[0.951]}\end{array}$ \\
\hline $\mathrm{R} \& \mathrm{D}$ expenditures/assets & $\begin{array}{c}1.191 \\
{[0.089]^{*}}\end{array}$ & $\begin{array}{c}1.186 \\
{[0.070]^{*}}\end{array}$ & $\begin{array}{l}2.326 \\
{[0.000]^{* * *}}\end{array}$ & $\begin{array}{l}2.042 \\
{[0.000]^{* * * *}}\end{array}$ \\
\hline Capital expenditures/assets & $\begin{array}{c}0.042 \\
{[0.913]}\end{array}$ & $\begin{array}{c}-0.017 \\
{[0.962]}\end{array}$ & $\begin{array}{c}0.159 \\
{[0.112]}\end{array}$ & $\begin{array}{l}0.303 \\
{[0.003]^{* * * *}}\end{array}$ \\
\hline Leverage ratio & $\begin{array}{c}0.832 \\
{[0.023]^{* *}}\end{array}$ & $\begin{array}{c}0.815 \\
{[0.017]^{* * *}}\end{array}$ & $\begin{array}{l}0.256 \\
{[0.000]^{* * *}}\end{array}$ & $\begin{array}{l}0.245 \\
{[0.000]^{* * *}}\end{array}$ \\
\hline S\&P 500 volatility & & & 0.433 & 0.240 \\
\hline$N$ & 21,832 & 21,832 & 22,426 & 22,426 \\
\hline Year fixed effects & Yes & Yes & Yes & Yes \\
\hline Hansen statistics & 0.073 & 0.246 & & \\
\hline$(P$-value $)$ & 0.9641 & 0.8843 & & \\
\hline
\end{tabular}

Note: $P$-values in brackets.

* $P<0.10$. 
With the implementation of FAS123R in 2006, public corporations were required to expense stock option grants at their fair market values. Our last instrument is the accounting cost of implementing FAS123R (FAS cost), which is proxied with the ratio of the estimated market value of annual CEO option grants to reported net income. This ratio measures how much the reported net income of a firm would decline if stock option grants were expensed at their fair value. Prior to FAS123R, firms with high accounting costs granted more options, but following the rule change, these firms reduced stock option grants more because they would have had a larger accounting impact on their profitability measures (Hayes, Lemmon, and Qiu, 2012). This non-uniform response to the regulation implies a positive relationship between vega and accounting cost of FAS 123R. However, we do not expect FAS cost to affect firm risk. In fact, Hayes, Lemmon, and Qiu (2012) show that the passage of FAS 123R has not been accompanied by a similar decline in firm risk.

A concern in the context of our study is that the interaction of delta and the macroeconomic state may be endogenous with respect to risk taking. The 2SLS estimation is also helpful in alleviating this additional endogeneity concern because the interactions of endogenous variables with an exogenous variable are treated as endogenous in the estimation. The interactions of instruments for the endogenous variables with the exogenous variable serve as valid instruments (Bun and Harrison, 2014; Wooldridge, 2002, pp. 121-122). Therefore, aside from delta and vega, we also treat the interactions of delta and vega with the macroeconomic state measure as endogenous in our first-stage regressions. Additionally, we include the interactions of the surplus cash, tax-loss carry forward indicator, and the FAS cost with the macroeconomic state measure in our list of instruments.

The instrumental variable estimation involves 2SLS method, where we first regress delta and vega on the instruments and the exogenous controls. Then, we regress the total firm risk on the predicted values of delta and vega. Our first-stage regression results are in line with the prior research (Coles, Daniel, and Naveen, 2006; Armstrong and Vashishtha, 2012). ${ }^{2}$ We conduct the Hansen's test of overidentifying restrictions to see whether the exclusion restriction holds. We find that the J-statistics associated with the test are statistically insignificant at the $10 \%$ level. Therefore, the assumption that the instruments are exogenous is unlikely to be violated. In addition, the partial F-statistics suggest that as a group, our instruments have a significant explanatory power in both the delta and vega regressions at the $1 \%$ level and confirm that our results are not subject to the weak instrument bias.

In Columns 1 and 2 of Table VI, we present the second-stage results. We find a statistically significant positive coefficient on delta and its interaction term with GDP growth variable. The 2SLS estimation results confirm the robustness of our main finding to the endogenous treatment of contract design, as well as the endogeneity of the interaction of delta and the macroeconomic state with respect to risk taking.

\section{2.b. Simultaneous equations regressions}

We also estimate firm risk, delta, and vega in a simultaneous regression framework in order to address the concern that the executive compensation contracts and firm risk might be jointly determined. We follow the same specification as in the instrumental variables estimation for delta and vega. Simultaneous equations require an additional variable in each equation that is unique to that equation for identification. For delta and vega equations, we use the median delta and vega values in the firm's industry since compensation structures

2 Unreported for brevity. Available upon request. 
vary across industries. For firm risk, we include the volatility of the S\&P 500 index. All dependent variables in the system are explicitly taken to be endogenous and are treated as correlated with the disturbances in the system's equations. The system is estimated by the 3SLS method, which is the most efficient estimation technique for the simultaneous systems (Schmidt, 1976; Greene, 2003).

In Columns 3 and 4 of Table VI, we present the results for interaction of the macroeconomic state with managerial performance incentives for total risk and firm-specific risk equations respectively. Once again, in line with the predictions of our hypothesis, we obtain a statistically significant positive coefficient on the interaction terms. These results confirm the main finding in the article-the relationship between managerial performance incentives and firm risk depends on the underlying macroeconomic state and gets stronger (weaker) as the economy expands (contracts).

\subsection{Firm Value Declines or Macroeconomic State?}

The main result in this article may not be related to the underlying macroeconomic environment per se, but to the general declines in firm values that accompany the recessions. If the impact of macroeconomic state disappears once we control for firm value declines, then the results we present are merely an artifact of decreasing stock values during recessions and would undermine our hypothesis that delta-risk relationship is pro-cyclical. Here, we address this concern with additional tests.

We start with investigating whether delta-risk relationship is weaker when firm valuations decrease. To this end, we separate the firms into two subsamples: (i) Firms whose stock values decline over a fiscal year and (ii) firms whose stock values increase or stay the same over a fiscal year. We create an indicator variable (Value Decline) that takes the value one for negative return firms and zero otherwise. We perform a split-sample analysis by estimating the baseline regression (Equation (1)) for the two groups separately. We then augment the baseline regression equation by adding the "Value Decline" indicator and its interactions with delta and vega to formally test whether there is a significant difference in the relationship between pay-for-performance sensitivity and firm risk when the firm value declines.

We present the results in Table VII. The results indicate that the firms that experience value decreases over a fiscal year tend to increase firm risk whereas firms that experience value increases over a fiscal year tend to decrease firm risk. For firms with declining values, the coefficient on delta is significant and positive (Column 1), whereas for firms with increasing values the coefficient on delta is significant and negative (Column 2). The coefficient on the interaction term (Value Decline * Delta) is 0.031 and significant at the $1 \%$ level (Column 3). This difference-in-difference analysis implies that a $1 \%$ increase in delta is associated with an additional $0.031 \%$ increase in firm risk for firms that experience declines in their stock valuations compared with firms whose values do not decrease.

This finding is counterintuitive because the theory predicts that a reduction in a firm's stock price would have a depressive effect on the manager's risk-taking incentives. A possible explanation might be related to how managers are evaluated for their performancemanagers are assessed annually and respond to the changes in firm performance regardless of their incentives. In response to poor midyear performance, increasing risk may increase the chance of catching up with the peers (Brown, Harlow, and Starks, 1996; Kempf, Ruenzi, and Thiele, 2009), hence contrary to the theoretical prediction, managers of firms 
Table VII. Effect of macroeconomic environment versus firm values

This table presents the results for the estimation of Equation (1) and Equation (2) in the text for firms with declining and increasing valuations. The dependent variable is firm risk, calculated as the logarithm of the annualized variance of the daily stock returns. The main variables of interest is the interaction of logarithm of the lagged value of delta with the value decline indicator (Column 3), and the interaction of logarithm of the lagged value of delta with the NBER recession dummy and value decline indicator (Column 6). The definitions of the variables are provided in Appendix A. All regressions control for year fixed effects. Robust standard errors are clustered at year-firm level. $P$-values are provided in brackets. ${ }^{*},{ }^{*}$, and ${ }^{* * *}$ mark the $10 \%$, $5 \%$, and $1 \%$ statistical significance for the estimated coefficients.

\begin{tabular}{|c|c|c|c|c|c|c|}
\hline & \multirow[b]{2}{*}{$\begin{array}{l}\text { Subsample: } \\
\text { value } \\
\text { decline }\end{array}$} & \multirow[b]{2}{*}{$\begin{array}{l}\text { Subsample: } \\
\text { value } \\
\text { increase }\end{array}$} & \multirow[b]{2}{*}{$\begin{array}{l}\text { Full } \\
\text { sample }\end{array}$} & \multicolumn{3}{|c|}{ Interactions with the recession dummy } \\
\hline & & & & $\begin{array}{l}\text { Subsample: } \\
\text { negative } \\
\text { return }\end{array}$ & $\begin{array}{l}\text { Subsample: } \\
\text { positive } \\
\text { return }\end{array}$ & $\begin{array}{l}\text { Full } \\
\text { sample }\end{array}$ \\
\hline $\log \left(\right.$ Delta $\left._{-1}\right)$ & $\begin{array}{l}0.024 \\
{[0.042]^{* *}}\end{array}$ & $\begin{array}{l}-0.028 \\
{[0.000]^{* * *}}\end{array}$ & $\begin{array}{l}-0.023 \\
{[0.001]^{* * * *}}\end{array}$ & $\begin{array}{l}0.042 \\
{[0.000]^{* * *}}\end{array}$ & $\begin{array}{c}0.004 \\
{[0.539]}\end{array}$ & $\begin{array}{c}0.008 \\
{[0.189]}\end{array}$ \\
\hline $\log \left(\operatorname{Vega}_{-1}\right)$ & $\begin{array}{l}-0.052 \\
{[0.000]^{* * *}}\end{array}$ & $\begin{array}{l}-0.040 \\
{[0.000]^{* * *}}\end{array}$ & $\begin{array}{l}-0.056 \\
{[0.000]^{* * *}}\end{array}$ & $\begin{array}{l}-0.046 \\
{[0.000]^{* * *}}\end{array}$ & $\begin{array}{l}-0.040 \\
{[0.000]^{* * *}}\end{array}$ & $\begin{array}{l}-0.050 \\
{[0.000]^{* * * *}}\end{array}$ \\
\hline Negative return & & & $\begin{array}{l}0.087 \\
{[0.009]^{* * *}}\end{array}$ & & & $\begin{array}{l}0.100 \\
{[0.003]^{* * *}}\end{array}$ \\
\hline $\log \left(\right.$ Delta $\left._{-1}\right) *$ Value decline & & & $\begin{array}{l}0.031 \\
{[0.000]^{* * *}}\end{array}$ & & & $\begin{array}{l}0.019 \\
{[0.005]^{* * *}}\end{array}$ \\
\hline $\log \left(\right.$ Vega $\left._{-1}\right) *$ Value decline & & & $\begin{array}{l}0.027 \\
{[0.000]^{* * *}}\end{array}$ & & & $\begin{array}{l}0.024 \\
{[0.000]^{* * *}}\end{array}$ \\
\hline NBER recession dummy & & & & $\begin{array}{l}1.080 \\
{[0.000]^{* * *}}\end{array}$ & $\begin{array}{l}1.131 \\
{[0.000] * * *}\end{array}$ & $\begin{array}{l}1.078 \\
{[0.000]^{* * *}}\end{array}$ \\
\hline $\log \left(\right.$ Delta $\left._{-1}\right) *$ Recession & & & & $\begin{array}{l}-0.050 \\
{[0.001]^{* * *}}\end{array}$ & $\begin{array}{l}-0.082 \\
{[0.000]^{* * *}}\end{array}$ & $\begin{array}{l}-0.075 \\
{[0.000]^{* * *}}\end{array}$ \\
\hline $\log \left(\operatorname{Vega}_{-1}\right) *$ Recession & & & & $\begin{array}{r}-0.019 \\
{[0.182]}\end{array}$ & $\begin{array}{c}0.000 \\
{[0.971]}\end{array}$ & $\begin{array}{r}-0.001 \\
{[0.906]}\end{array}$ \\
\hline Value decline * Recession & & & & & & $\begin{array}{c}0.028 \\
{[0.707]}\end{array}$ \\
\hline $\begin{array}{l}\log \left(\text { Delta }_{-1}\right) * \text { Recession * } \\
\text { Value decline }\end{array}$ & & & & & & $\begin{array}{c}0.024 \\
{[0.110]}\end{array}$ \\
\hline $\begin{array}{l}\log \left(\text { Vega }_{-1}\right) * \text { Recession } * \\
\text { Value decline }\end{array}$ & & & & & & $\begin{array}{c}-0.016 \\
{[0.197]}\end{array}$ \\
\hline $\left.\log _{(\text {Cash compensation }}{ }_{-1}\right)$ & $\begin{array}{c}-0.011 \\
{[0.692]}\end{array}$ & $\begin{array}{l}-0.076 \\
{[0.000]^{* * *}}\end{array}$ & $\begin{array}{l}-0.050 \\
{[0.003]^{* * *}}\end{array}$ & $\begin{array}{l}-0.085 \\
{[0.001]^{* * *}}\end{array}$ & $\begin{array}{l}-0.122 \\
{[0.000]^{* * *}}\end{array}$ & $\begin{array}{l}-0.106 \\
{[0.000]^{* * *}}\end{array}$ \\
\hline CEO tenure & $\begin{array}{l}-0.005 \\
{[0.045]^{* *}}\end{array}$ & $\begin{array}{l}-0.003 \\
{[0.095]^{*}}\end{array}$ & $\begin{array}{l}-0.004 \\
{[0.009]^{* * * *}}\end{array}$ & $\begin{array}{c}-0.003 \\
{[0.189]}\end{array}$ & $\begin{array}{c}-0.003 \\
{[0.115]}\end{array}$ & $\begin{array}{l}-0.003 \\
{[0.047]^{* * *}}\end{array}$ \\
\hline $\log ($ Sales $)$ & $\begin{array}{l}-0.057 \\
{[0.065]^{*}}\end{array}$ & $\begin{array}{l}-0.080 \\
{[0.000]^{* * *}}\end{array}$ & $\begin{array}{l}-0.062 \\
{[0.002]^{* * * *}}\end{array}$ & $\begin{array}{l}-0.127 \\
{[0.000]^{* * * *}}\end{array}$ & $\begin{array}{l}-0.130 \\
{[0.000]^{* * *}}\end{array}$ & $\begin{array}{l}-0.125 \\
{[0.000]^{* * *}}\end{array}$ \\
\hline Market-to-book & $\begin{array}{l}-0.100 \\
{[0.000]^{* * *}}\end{array}$ & $\begin{array}{l}0.077 \\
{[0.000]^{* * *}}\end{array}$ & $\begin{array}{l}0.033 \\
{[0.000]^{* * *}}\end{array}$ & $\begin{array}{c}-0.041 \\
{[0.053]^{*}}\end{array}$ & $\begin{array}{l}0.114 \\
{[0.000] * * *}\end{array}$ & $\begin{array}{l}0.070 \\
{[0.000]^{* * *}}\end{array}$ \\
\hline R\&D expenditures/assets & $\begin{array}{l}0.520 \\
{[0.032]^{* *}}\end{array}$ & $\begin{array}{c}0.477 \\
{[0.137]}\end{array}$ & $\begin{array}{l}0.415 \\
{[0.032]^{* *}}\end{array}$ & $\begin{array}{c}0.234 \\
{[0.249]}\end{array}$ & $\begin{array}{c}0.100 \\
{[0.750]}\end{array}$ & $\begin{array}{c}0.181 \\
{[0.288]}\end{array}$ \\
\hline Capital expenditures/assets & $\begin{array}{c}-0.016 \\
{[0.961]}\end{array}$ & $\begin{array}{l}-1.185 \\
{[0.000]^{* * *}}\end{array}$ & $\begin{array}{l}-0.573 \\
{[0.006]^{* * *}}\end{array}$ & $\begin{array}{c}0.309 \\
{[0.264]}\end{array}$ & $\begin{array}{l}-0.628 \\
{[0.003]^{* * *}}\end{array}$ & $\begin{array}{c}-0.153 \\
{[0.395]}\end{array}$ \\
\hline Debt/assets & $\begin{array}{l}0.690 \\
{[0.000]^{* * *}}\end{array}$ & $\begin{array}{l}0.696 \\
{[0.000]^{* * *}}\end{array}$ & $\begin{array}{l}0.668 \\
{[0.000]^{* * *}}\end{array}$ & $\begin{array}{l}0.541 \\
{[0.001]^{* * *}}\end{array}$ & $\begin{array}{c}0.600 \\
{[0.000]^{* * *}}\end{array}$ & $\begin{array}{c}0.554 \\
{[0.000]^{* * *}}\end{array}$ \\
\hline
\end{tabular}


Table VII. Continued

\begin{tabular}{|c|c|c|c|c|c|c|}
\hline & \multirow[b]{2}{*}{$\begin{array}{l}\text { Subsample: } \\
\text { value } \\
\text { decline }\end{array}$} & \multirow[b]{2}{*}{$\begin{array}{l}\text { Subsample: } \\
\text { value } \\
\text { increase }\end{array}$} & \multirow[b]{2}{*}{$\begin{array}{l}\text { Full } \\
\text { sample }\end{array}$} & \multicolumn{3}{|c|}{ Interactions with the recession dummy } \\
\hline & & & & $\begin{array}{l}\text { Subsample: } \\
\text { negative } \\
\text { return }\end{array}$ & $\begin{array}{l}\text { Subsample: } \\
\text { positive } \\
\text { return }\end{array}$ & $\begin{array}{l}\text { Full } \\
\text { sample }\end{array}$ \\
\hline Constant & $\begin{array}{l}-0.954 \\
{[0.000]^{* * *}}\end{array}$ & $\begin{array}{l}-0.838 \\
{[0.000]^{* * *}}\end{array}$ & $\begin{array}{l}-0.985 \\
{[0.000]^{* * *}}\end{array}$ & $\begin{array}{c}-0.375 \\
{[0.110]}\end{array}$ & $\begin{array}{l}-0.548 \\
{[0.001]^{* * *}}\end{array}$ & $\begin{array}{l}-0.550 \\
{[0.000]^{* * *}}\end{array}$ \\
\hline$N$ & 8,127 & 11,687 & 19,814 & 8,127 & 11,687 & 19,814 \\
\hline Year fixed effects & Yes & Yes & Yes & Yes & Yes & Yes \\
\hline Firm-manager fixed effects & Yes & Yes & Yes & Yes & Yes & Yes \\
\hline$R$-squared & 0.033 & 0.065 & 0.088 & 0.230 & 0.252 & 0.271 \\
\hline Adjusted $R$-squared & 0.031 & 0.065 & 0.087 & 0.228 & 0.251 & 0.270 \\
\hline
\end{tabular}

Note: $P$-values in brackets.

$* P<0.10, * * P<0.05, * * P<0.01$.

that experience stock price declines may choose to increase firm risk instead of reducing it. This finding might also be related to the fact that the wealth effect is particularly difficult to capture due to the variations in CEOs' outside wealth, which we cannot directly measure in the US setting because of lack of data. Neyland (2012) gets around this problem by using spousal divorce as a proxy for a negative shock to a CEO's outside wealth. He finds that firm risk decreases during the years of spousal divorce and attributes this result to the increase in managerial risk aversion as a response to the decline in the wealth of the executives following divorce.

Next, we look at firm value effects during recessions. We note that during recessions, 2,120 firms in our sample experienced value declines, and 1,978 firms experienced value increases. Therefore, we have variation in firm performance during fiscal years that coincide with NBER recession dates. If the main result we present in the article is solely related to declines in firm values and not related to the macroenvironment, then the weakening of delta-risk relation should only be valid for firms that experience a decrease in value and not for firms whose value increase during recessions. To explore this possibility, in Columns 4 and 5, we perform a split-sample analysis by estimating Equation (2) for the two groups separately, and in Column 6, we augment our main regression equation (Equation (2)) by adding the negative return dummy and its interactions with delta, vega, and the recession indicator along with the triple interaction term (Delta * Value Decline * Recession). This specification enables us to distinguish between firms whose values decrease in recessions and firms whose values increase during recessions.

The results show that the effect of delta on firm risk is negative during recessions for both types of firms (Columns 4 and 5). It is especially reassuring to see that the delta-risk relationship weakens even for firms that experience value increases during recessions (Column 5). Finally, we find that the coefficient on the triple interaction term is insignificant at the $10 \%$ level (Column 6$).{ }^{3}$ Recessions seem to have a significant effect on risk-taking decisions regardless of the direction of firm value changes. These results are robust to

3 This finding is also in line with Guiso, Sapienza, and Zingales (2014), who show that even the people who do not face any financial losses exhibit higher levels of risk aversion during recessions. 
employing the continuous measures of macroeconomic state instead of the recession indicator and also to using firm-specific risk as the dependent variable. We also repeat the analyses using declines in firm values that are orthogonal to macroeconomic conditions and obtain the same results.

The finding that the delta-risk relationship responds similarly to macroeconomic recessions regardless of whether the firm's stock price increases or decreases during the recession suggests that a shift in risk aversion is the most likely explanation of our main result. In addition, the main finding in the article is not valid for those firms that operate in recession-proof industries. ${ }^{4}$ This result is in line with our main hypothesis because managers in recession-proof industries would not expect recessions to impact their firms significantly, hence their risk aversion would be less sensitive to the underlying macroeconomic environment.

\subsection{CEO Control}

The main source of pro-cyclicality between delta and firm risk that we document in this article is the agency problem caused by the differences in risk attitudes of managers and shareholders. Such an agency problem would be more pronounced when a manager has more control over the firm's resources. These managers will be able to influence firms' business decisions to a greater extent and therefore affect firm risk more. Hence, in this section, we focus on two variables that are correlated with managerial control: CEO tenure and product market competition.

CEO tenure has been widely used as a proxy for CEO control in the literature (e.g., in Fahlenbrach, 2009; Bebchuk, Grinstein, and Peyer, 2010; Chava, Kumar, and Warga, 2010; Ferreira, Ferreira, and Raposo, 2011; Agrawal and Nasser, 2012). Boards' control over a CEOs' actions are expected to decline as CEOs become more seasoned in the firm. Baker and Gompers (2003) show that the representation of independent outsiders on the board decreases with the tenure of the CEO. A CEO with a longer tenure is also more likely to capture the board of directors because directors that are appointed by a CEO exert less control over him/her (Shivdasani and Yermack, 1999; Baker and Gompers, 2003; Morse, Nanda, and Seru, 2011; Coles, Daniel, and Naveen, 2014). Coles, Daniel, and Naveen (2014) develop a measure of "board co-option"-the percentage of the board members that are appointed during a CEOs tenure and show that board co-option increases with tenure, while the monitoring effectiveness of the board of directors decreases with co-option. ${ }^{5}$ As boards' monitoring effectiveness decline, the CEOs would have a larger effect over company policies and therefore exert more control over firm risk. For instance, CEOs influence R\&D spending to suit their own preferences as their tenure increase (Baker and Mueller, 2002). Similarly, more seasoned CEOs (with a tenure exceeding 3 years) tend to have more power over the board of directors (Pan, Wang, and Weisbach, 2014) and the CEO tenure effect exists regardless of the macroeconomic conditions or the industry conditions in which the CEO takes office.

4 Recession proof industries are oil and gas, food manufacturing, beverage, tobacco, mining, utilities, and healthcare. These are the industries that are characterized with market betas that are lower than 1 (Hong and Kacperczyk, 2009). Results are available upon request.

5 We also find that CEOs that are monitored by more co-opted boards are more able to decrease firm risk during recessions. We thank Lalitha Naveen for sharing the board co-option data with us. 
We divide the firms into two subsamples: (i) firms whose CEOs have a tenure exceeding 3 years and (ii) firms whose CEOs have a tenure less than or equal to 3 years. We create a dummy variable that takes the value one for firms that are managed by long-tenured CEOs and zero otherwise. We test whether the delta-risk relationship is weaker during recessions for firms with more seasoned CEOs. In line with our prediction, we find that the effect of delta on firm risk during recessions is significantly negative for those firms with seasoned CEOs (Table VIII, Column 1). Although the coefficient is also negative for firms with short tenured CEOs, the effect is statistically insignificant at the $10 \%$ level. The coefficient on the triple interaction term is negative $(-0.045)$ and significant at the $10 \%$ level (Column 3) implying that firms with seasoned CEOs tend to reduce firm risk during recessions more compared with those firms that are run by short tenure CEOs.

Product market competition acts as an important external governance device that limits the managers' ability to use firms' resources according to their own personal preferences. In fact, there is evidence that product market competition may be a substitute for internal governance mechanisms (Giroud and Mueller, 2010; Chhaochharia et al., 2012). The recent empirical literature indicates that product market competition reduces managerial indiscipline (Giroud and Mueller, 2010, 2011; Grullon and Michaely, 2012) and improves management practices (Bloom and Van Reenen, 2007; Masulis, Wang, and Xie, 2007; Bloom et al., 2015) show that product market competition is a disciplinary force on managers, leading to higher corporate payouts. As these studies show, high product market competition tends to be a disciplining force on managers. Therefore, we expect these managers to be less able to adjust firms' operations to alter firm risk according to their own preferences.

To identify the instances of low product market competition, we use the HerfindahlHirschman index (HHI) of industry concentration developed by Hoberg and Phillips (2014) based on a group of competitor companies determined by pairwise similarity scores of product descriptions reported in 10-K reports. Overall, high HHI scores indicate lower product market competition and we expect the delta-risk relationship to be more sensitive to the changes in the macroeconomic environment in firms with lower HHI scores. ${ }^{6}$ We divide the firms into two subsamples according to their HHIs: (i) firms whose HHI scores are above the sample median and (ii) firms whose HHI scores are less than or equal to the median. We create an indicator variable that takes the value one for high HHI firms and zero otherwise. We perform a split-sample analysis by running Equation (2) for the two groups separately. The effect of delta on firm risk during recessions is significantly negative for firms that face limited product market competition (Table VIII, Column 4). The coefficient for firms that face significant product market competition is statistically insignificant at the $10 \%$ level. We also augment the regression equation by adding the high HHI dummy, its interactions with delta, vega, the recession indicator along with the triple interaction term (Delta * high HHI * recession). The coefficient on the triple interaction term is negative $(-0.041)$ and significant at the $1 \%$ level (Column 6) implying that firms that face less competition tend to reduce firm risk more during recessions in response to a given level of delta compared with those firms that operate in a more competitive environment.

Overall, our analyses based on CEO tenure and product market competition as proxies for CEO power suggest that the delta-risk relationship becomes more sensitive to the

6 We also check our results using the product market fluidity index that measures the emerging product market threats from other firms (Hoberg, Phillips, and Prabhala, 2014) as an alternative measure for product market competition. Results are unchanged. 
Table VIII. Managerial control

This table presents the results for the estimation of Equation (2) in the text for firms with higher and lower levels of managerial control. The dependent variable is firm risk, calculated as the logarithm of the annualized variance of the daily stock returns. Managerial control is proxied by CEO tenure in Columns 1-3 and by HHI in columns. The main variables of interest are the interactions of logarithm of the lagged value of delta with the NBER recession dummy and managerial control measures (Columns 3,6, and 9). The definitions of the variables are provided in Appendix A. All regressions control for year fixed effects. Robust standard errors are clustered at firm level. $P$-values are provided in brackets. ${ }^{*},{ }^{* *}$, and ${ }^{* * *}$ mark the $10 \%, 5 \%$, and $1 \%$ statistical significance for the estimated coefficients.

\begin{tabular}{|c|c|c|c|c|c|c|}
\hline \multirow{2}{*}{$\begin{array}{l}\text { Managerial control } \\
\text { measured by: }\end{array}$} & \multicolumn{3}{|l|}{ CEO tenure } & \multicolumn{3}{|l|}{$\mathrm{HHI}$} \\
\hline & $\begin{array}{l}\text { Long CEO } \\
\text { tenure }\end{array}$ & $\begin{array}{l}\text { Short CEO } \\
\text { tenure }\end{array}$ & $\begin{array}{l}\text { Full } \\
\text { sample }\end{array}$ & $\begin{array}{l}\text { High } \\
\text { HHI }\end{array}$ & $\begin{array}{l}\text { Low } \\
\text { HHI }\end{array}$ & $\begin{array}{l}\text { Full } \\
\text { sample }\end{array}$ \\
\hline $\log \left(\right.$ Delta $\left._{-1}\right)$ & $\begin{array}{l}0.022 \\
{[0.000]^{* * *}}\end{array}$ & $\begin{array}{c}0.038 \\
{[0.077]^{*}}\end{array}$ & $\begin{array}{l}0.037 \\
{[0.000]^{* * *}}\end{array}$ & $\begin{array}{c}0.018 \\
{[0.035]^{* *}}\end{array}$ & $\begin{array}{l}0.029 \\
{[0.000]^{* * *}}\end{array}$ & $\begin{array}{l}0.027 \\
{[0.000]^{* * *}}\end{array}$ \\
\hline $\log \left(\operatorname{Vega}_{-1}\right)$ & $\begin{array}{l}-0.047 \\
{[0.000]^{* * *}}\end{array}$ & $\begin{array}{l}-0.049 \\
{[0.023]^{* *}}\end{array}$ & $\begin{array}{l}-0.055 \\
{[0.000]^{* * *}}\end{array}$ & $\begin{array}{l}-0.050 \\
{[0.000]^{* * *}}\end{array}$ & $\begin{array}{l}-0.044 \\
{[0.000]^{* * *}}\end{array}$ & $\begin{array}{l}-0.052 \\
{[0.000]^{* * *}}\end{array}$ \\
\hline NBER recession dummy & $\begin{array}{l}0.269 \\
{[0.000]^{* * *}}\end{array}$ & $\begin{array}{c}0.238 \\
{[0.166]}\end{array}$ & $\begin{array}{l}0.198 \\
{[0.035]^{* *}}\end{array}$ & $\begin{array}{l}0.296 \\
{[0.000]^{* * *}}\end{array}$ & $\begin{array}{l}0.204 \\
{[0.016]^{* * *}}\end{array}$ & $\begin{array}{l}0.162 \\
{[0.019]^{* *}}\end{array}$ \\
\hline $\log \left(\right.$ Delta $\left._{-1}\right) *$ Recession & $\begin{array}{l}-0.020 \\
{[0.037]^{* *}}\end{array}$ & $\begin{array}{c}-0.023 \\
{[0.603]}\end{array}$ & $\begin{array}{c}0.019 \\
{[0.446]}\end{array}$ & $\begin{array}{l}-0.037 \\
{[0.003]^{* * * *}}\end{array}$ & $\begin{array}{c}-0.003 \\
{[0.804]}\end{array}$ & $\begin{array}{r}-0.001 \\
{[0.957]}\end{array}$ \\
\hline $\log \left(\right.$ Vega $\left._{-1}\right) *$ Recession & $\begin{array}{c}-0.004 \\
{[0.638]}\end{array}$ & $\begin{array}{c}-0.039 \\
{[0.382]}\end{array}$ & $\begin{array}{l}-0.055 \\
{[0.013]^{* *}}\end{array}$ & $\begin{array}{c}0.011 \\
{[0.350]}\end{array}$ & $\begin{array}{c}-0.009 \\
{[0.409]}\end{array}$ & $\begin{array}{c}-0.004 \\
{[0.668]}\end{array}$ \\
\hline Managerial control & & & $\begin{array}{l}0.070 \\
{[0.037]^{* *}}\end{array}$ & & & $\begin{array}{c}0.028 \\
{[0.397]}\end{array}$ \\
\hline Managerial control * Recession & & & $\begin{array}{c}0.099 \\
{[0.304]}\end{array}$ & & & $\begin{array}{l}0.214 \\
{[0.008]^{* * *}}\end{array}$ \\
\hline $\begin{array}{c}\log \left(\text { Delta }_{-1}\right) * \text { Recession } * \\
\text { Managerial control }\end{array}$ & & & $\begin{array}{l}-0.045 \\
{[0.083]^{*}}\end{array}$ & & & $\begin{array}{c}-0.010 \\
{[0.127]}\end{array}$ \\
\hline $\log \left(\right.$ Delta $\left._{-1}\right) *$ Managerial control & & & $\begin{array}{l}-0.017 \\
{[0.095]^{*}}\end{array}$ & & & $\begin{array}{l}-0.042 \\
{[0.011]^{* *}}\end{array}$ \\
\hline $\log \left(\right.$ Vega $\left._{-1}\right) *$ Managerial control & & & $\begin{array}{c}0.009 \\
{[0.349]}\end{array}$ & & & $\begin{array}{l}0.014 \\
{[0.039]^{* *}}\end{array}$ \\
\hline $\begin{array}{l}\log \left(\text { Vega }_{-1}\right) * \text { Recession } \\
\text { Managerial control }\end{array}$ & & & $\begin{array}{l}0.057 \\
{[0.014]^{* *}}\end{array}$ & & & $\begin{array}{c}0.004 \\
{[0.757]}\end{array}$ \\
\hline $\log _{(\text {Cash compensation }}$ com $\left._{1}\right)$ & $\begin{array}{l}-0.055 \\
{[0.000]^{* * *}}\end{array}$ & $\begin{array}{c}-0.050 \\
{[0.136]}\end{array}$ & $\begin{array}{l}-0.060 \\
{[0.000]^{* * *}}\end{array}$ & $\begin{array}{l}-0.086 \\
{[0.000]^{* * *}}\end{array}$ & $\begin{array}{l}-0.033 \\
{[0.022]^{* *}}\end{array}$ & $\begin{array}{l}-0.057 \\
{[0.000] * * *}\end{array}$ \\
\hline CEO tenure & $\begin{array}{c}0.012 \\
{[0.189]}\end{array}$ & $\begin{array}{l}0.067 \\
{[0.001]^{* * *}}\end{array}$ & $\begin{array}{c}0.012 \\
{[0.185]}\end{array}$ & $\begin{array}{c}0.008 \\
{[0.692]}\end{array}$ & $\begin{array}{c}0.017 \\
{[0.125]}\end{array}$ & $\begin{array}{c}0.013 \\
{[0.133]}\end{array}$ \\
\hline $\log ($ Sales $)$ & $\begin{array}{l}-0.101 \\
{[0.000] * * *}\end{array}$ & $\begin{array}{l}-0.187 \\
{[0.011]^{* *}}\end{array}$ & $\begin{array}{l}-0.106 \\
{[0.000]^{* * *}}\end{array}$ & $\begin{array}{l}-0.131 \\
{[0.000]^{* * *}}\end{array}$ & $\begin{array}{l}-0.095 \\
{[0.000]^{* * *}}\end{array}$ & $\begin{array}{l}-0.109 \\
{[0.000]^{* * *}}\end{array}$ \\
\hline Market-to-book & $\begin{array}{c}-0.006 \\
{[0.210]}\end{array}$ & $\begin{array}{l}-0.055 \\
{[0.001]^{* * *}}\end{array}$ & $\begin{array}{c}-0.004 \\
{[0.394]}\end{array}$ & $\begin{array}{l}-0.025 \\
{[0.003]^{* * *}}\end{array}$ & $\begin{array}{r}-0.004 \\
{[0.550]}\end{array}$ & $\begin{array}{c}-0.004 \\
{[0.407]}\end{array}$ \\
\hline R\&D expenditures/assets & $\begin{array}{c}0.282 \\
{[0.021]^{* *}}\end{array}$ & $\begin{array}{c}-0.110 \\
{[0.795]}\end{array}$ & $\begin{array}{l}0.284 \\
{[0.009]^{* * *}}\end{array}$ & $\begin{array}{c}0.088 \\
{[0.521]}\end{array}$ & $\begin{array}{c}0.321 \\
{[0.021]^{* *}}\end{array}$ & $\begin{array}{l}0.272 \\
{[0.012]^{* *}}\end{array}$ \\
\hline Capital expenditures/assets & $\begin{array}{l}0.339 \\
{[0.007]^{* * *}}\end{array}$ & $\begin{array}{c}0.003 \\
{[0.994]}\end{array}$ & $\begin{array}{l}0.402 \\
{[0.000]^{* * *}}\end{array}$ & $\begin{array}{c}0.486 \\
{[0.018]^{* *}}\end{array}$ & $\begin{array}{l}0.402 \\
{[0.005]^{* * *}}\end{array}$ & $\begin{array}{l}0.396 \\
{[0.000]^{* * *}}\end{array}$ \\
\hline Debt/assets & $\begin{array}{l}0.409 \\
{[0.000]^{* * *}}\end{array}$ & $\begin{array}{c}0.287 \\
{[0.121]}\end{array}$ & $\begin{array}{l}0.396 \\
{[0.000]^{* * *}}\end{array}$ & $\begin{array}{l}0.368 \\
{[0.000]^{* * *}}\end{array}$ & $\begin{array}{l}0.401 \\
{[0.000]^{* * * *}}\end{array}$ & $\begin{array}{l}0.397 \\
{[0.000]^{* * *}}\end{array}$ \\
\hline Constant & $\begin{array}{l}-1.111 \\
{[0.000]^{* * *}}\end{array}$ & $\begin{array}{c}-0.141 \\
{[0.785]}\end{array}$ & $\begin{array}{l}-1.089 \\
{[0.000]^{* * * *}}\end{array}$ & $\begin{array}{l}-0.718 \\
{[0.000]^{* * *}}\end{array}$ & $\begin{array}{l}-1.264 \\
{[0.000]^{* * *}}\end{array}$ & $\begin{array}{l}-1.058 \\
{[0.000]^{* * *}}\end{array}$ \\
\hline$N$ & 14,648 & 3,536 & 18,184 & 8,054 & 10,130 & 18,184 \\
\hline$R$-squared & 0.526 & 0.415 & 0.535 & 0.533 & 0.557 & 0.535 \\
\hline Adjusted $R$-squared & 0.525 & 0.410 & 0.534 & 0.531 & 0.556 & 0.534 \\
\hline
\end{tabular}

Note: $P$-values in brackets.

$* P<0.10, * * P<0.05, * * * P<0.01$. 
underlying macroeconomic environment when managers have more control over firm policies. When there is less discipline imposed by internal and/or external governance sources, CEOs can have a strong influence over firm risk and hence can reduce firm risk during recessions more in response to a given level of delta compared with firms that are run by less powerful CEOs.

\subsection{Dollar-Dollar Measure}

As standard in the literature (Core, 1999; Coles, Daniel, and Naveen, 2006; Low, 2009), we use the dollar-percentage measure of pay-for-performance sensitivity (i.e., dollar change in $\mathrm{CEO}$ wealth for a $1 \%$ change in the stock price). However, we recognize that the macroeconomic conditions may have a mechanical impact on the value of performance incentives. A $1 \%$ increase in stock price would automatically lead to a larger increase in the dollar value of performance incentives during expansions to the extent that stock prices are higher. To illustrate with an example, let's consider a CEO that holds 1,000 units of her firm's stock. If the price of the stock is $\$ 10$, a $1 \%$ increase in stock price will increase the CEO's firm-related wealth by $\$ 100$. If on the other hand, the stock value increases to $\$ 20$ due to a positive macroeconomic environment, then a $1 \%$ increase in stock price will increase the CEO's wealth by $\$ 200$. As this simple example illustrates, the dollar-percentage measure is positively related to the macroeconomic conditions due to the higher stock prices during macroeconomic expansions.

To address this concern, we have included the macroeconomic state as an explanatory variable in both the delta and vega equations when we conducted instrumental variables and simultaneous equations estimations. Here, we provide an additional robustness check. We calculate the dollar-dollar measure of delta (i.e., the dollar increase in CEO wealth as a result of a dollar increase in stock price) and use it in our regressions instead of the dollarpercentage measure. The dollar-dollar measure of performance incentives is not sensitive to the level of the underlying stock price, and therefore is not impacted by the underlying macroeconomic conditions. We present the results using the dollar-dollar sensitivity in Table IX. Our results remain unchanged.

\subsection{Clustering of Standard Errors}

Throughout the article, we cluster the standard errors at the firm level, allowing the residuals of a given firm to be correlated across years. In the context of this study, the error terms are also possibly cross-sectionally correlated since macroeconomic recessions are likely to affect all firms, albeit differently. With only a few recessions, the number of truly independent observations may be small, resulting in under-estimation of the standard errors.

Ideally, we would cluster the standard errors both at the firm level and at the year level, using two-way clustering as suggested by Petersen (2009) and Thompson (2011). However, we do not have sufficient number of observations to cluster on both dimensions. As an alternative, we have re-estimated Equation (3) by clustering standard errors at the industryyear level. This approach would partially alleviate the concern for cross-sectional correlation in the error terms, because the most obvious driver of clustering is the cross-sectional correlation in firm volatilities among firms in the same industry, since recessions are likely to affect firms in different industries differently. ${ }^{7}$ Our results are robust to this alternative form of clustering.

7 We thank the referee for this suggestion. 
Table IX. Dollar-dollar sensitivity as a measure for managerial performance incentives

This table presents the results for the estimation of Equation (2) in the text. The dependent variable is firm risk, calculated as the logarithm of the annualized variance of the daily stock returns. Delta in these regressions is measured as the dollar increase in CEO wealth as a result of a dollar increase in stock price. The main variable of interest is the interaction of logarithm of the lagged value of delta with the macroeconomic state measure. The definitions of the variables are provided in Appendix A. All regressions control for year fixed effects. Robust standard errors are clustered at firm level in regressions that control for industry fixed effects and at firm-manager level in regressions that control for firm-manager pair fixed effects. $P$-values are provided in brackets. ${ }^{*}, *$, and ${ }^{* *}$ mark the $10 \%, 5 \%$, and $1 \%$ statistical significance for the estimated coefficients.

\begin{tabular}{|c|c|c|c|c|c|c|}
\hline \multirow{2}{*}{$\begin{array}{l}\text { Macroeconomic } \\
\text { state is measured by: }\end{array}$} & \multicolumn{3}{|c|}{ NBER recession dummy } & \multicolumn{3}{|l|}{ GDP growth } \\
\hline & $\begin{array}{l}\text { Industry } \\
\text { fixed } \\
\text { effects }\end{array}$ & $\begin{array}{l}\text { Firm } \\
\text { fixed } \\
\text { effects }\end{array}$ & $\begin{array}{l}\text { Firm- } \\
\text { manager } \\
\text { fixed effects }\end{array}$ & $\begin{array}{l}\text { Industry } \\
\text { fixed } \\
\text { effects }\end{array}$ & $\begin{array}{l}\text { Firm } \\
\text { fixed } \\
\text { effects }\end{array}$ & $\begin{array}{l}\text { Firm- } \\
\text { manager } \\
\text { fixed effects }\end{array}$ \\
\hline $\log \left(\right.$ Delta $\left._{-1}\right)$ & $\begin{array}{c}0.0024 \\
{[0.507]}\end{array}$ & $\begin{array}{l}0.0144 \\
{[0.000]^{* * *}}\end{array}$ & $\begin{array}{l}0.0201 \\
{[0.000]^{* * *}}\end{array}$ & $\begin{array}{l}-0.009 \\
{[0.013]^{* *}}\end{array}$ & $\begin{array}{l}0.012 \\
{[0.003]^{* * *}}\end{array}$ & $\begin{array}{l}0.018 \\
{[0.000] * * *}\end{array}$ \\
\hline $\log \left(\operatorname{Vega}_{-1}\right)$ & $\begin{array}{l}-0.0320 \\
{[0.000] * * *}\end{array}$ & $\begin{array}{l}-0.0480 \\
{[0.000]^{* * *}}\end{array}$ & $\begin{array}{l}-0.0440 \\
{[0.000]^{* * *}}\end{array}$ & $\begin{array}{l}-0.030 \\
{[0.000]^{* * *}}\end{array}$ & $\begin{array}{l}-0.051 \\
{[0.000] * * *}\end{array}$ & $\begin{array}{l}-0.048 \\
{[0.000]^{* * *}}\end{array}$ \\
\hline Macroeconomy & $\begin{array}{l}0.1596 \\
{[0.000]^{* * *}}\end{array}$ & $\begin{array}{l}0.2075 \\
{[0.000]^{* * *}}\end{array}$ & $\begin{array}{l}0.2072 \\
{[0.000]^{* * *}}\end{array}$ & $\begin{array}{l}-0.077 \\
{[0.000]^{* * *}}\end{array}$ & $\begin{array}{l}-0.076 \\
{[0.000]^{* * *}}\end{array}$ & $\begin{array}{l}-0.079 \\
{[0.000]^{* * *}}\end{array}$ \\
\hline $\begin{array}{l}\log \left(\text { Delta }_{-1}\right) * \\
\quad \text { Macroeconomic state }\end{array}$ & $\begin{array}{l}-0.1492 \\
{[0.000]^{* * *}}\end{array}$ & $\begin{array}{l}-0.1145 \\
{[0.003]^{* * *}}\end{array}$ & $\begin{array}{l}-0.1245 \\
{[0.003]^{* * *}}\end{array}$ & $\begin{array}{l}0.058 \\
{[0.000]^{* * *}}\end{array}$ & $\begin{array}{l}0.025 \\
{[0.001]^{* * *}}\end{array}$ & $\begin{array}{l}0.029 \\
{[0.000]^{* * *}}\end{array}$ \\
\hline $\begin{array}{l}\log \left(\operatorname{Vega}_{-1}\right) * \\
\quad \text { Macroeconomic state }\end{array}$ & $\begin{array}{c}-0.3078 \\
{[0.088]^{*}}\end{array}$ & $\begin{array}{l}-0.4927 \\
{[0.002] * * *}\end{array}$ & $\begin{array}{l}-0.4636 \\
{[0.007] * * *}\end{array}$ & $\begin{array}{c}0.056 \\
{[0.108]}\end{array}$ & $\begin{array}{c}0.057 \\
{[0.062]^{*}}\end{array}$ & $\begin{array}{c}0.038 \\
{[0.216]}\end{array}$ \\
\hline $\log _{\left(\text {Cash } \text { compensation }_{-1}\right)}$ & $\begin{array}{l}-0.0892 \\
{[0.000]^{*} * *}\end{array}$ & $\begin{array}{l}-0.0741 \\
{[0.000]^{* * *}}\end{array}$ & $\begin{array}{l}-0.0594 \\
{[0.000]^{* * *}}\end{array}$ & $\begin{array}{l}-0.088 \\
{[0.000] * * *}\end{array}$ & $\begin{array}{l}-0.073 \\
{[0.000]^{* * *}}\end{array}$ & $\begin{array}{l}-0.059 \\
{[0.000]^{* * *}}\end{array}$ \\
\hline CEO tenure & $\begin{array}{c}-0.0006 \\
{[0.331]}\end{array}$ & $\begin{array}{l}-0.0021 \\
{[0.009]^{* * *}}\end{array}$ & $\begin{array}{c}0.0130 \\
{[0.141]}\end{array}$ & $\begin{array}{c}-0.001 \\
{[0.087]^{*}}\end{array}$ & $\begin{array}{l}-0.002 \\
{[0.002] * * *}\end{array}$ & $\begin{array}{c}0.013 \\
{[0.146]}\end{array}$ \\
\hline $\log ($ Sales $)$ & $\begin{array}{l}-0.1762 \\
{[0.000]^{* * *}}\end{array}$ & $\begin{array}{l}-0.1555 \\
{[0.000]^{* * *}}\end{array}$ & $\begin{array}{l}-0.1183 \\
{[0.000]^{* * *}}\end{array}$ & $\begin{array}{l}-0.165 \\
{[0.000]^{* * *}}\end{array}$ & $\begin{array}{l}-0.145 \\
{[0.000] * * *}\end{array}$ & $\begin{array}{l}-0.106 \\
{[0.000]^{* * *}}\end{array}$ \\
\hline Market-to-book & $\begin{array}{l}-0.0137 \\
{[0.000]^{* * *}}\end{array}$ & $\begin{array}{c}0.0073 \\
{[0.074]^{*}}\end{array}$ & $\begin{array}{c}-0.0041 \\
{[0.374]}\end{array}$ & $\begin{array}{l}-0.010 \\
{[0.011]^{* *}}\end{array}$ & $\begin{array}{l}0.009 \\
{[0.028]^{* *}}\end{array}$ & $\begin{array}{c}-0.003 \\
{[0.526]}\end{array}$ \\
\hline $\mathrm{R} \& \mathrm{D}$ expenditures/assets & $\begin{array}{l}1.8508 \\
{[0.000]^{* * *}}\end{array}$ & $\begin{array}{c}0.2409 \\
{[0.017]^{* *}}\end{array}$ & $\begin{array}{c}0.2704 \\
{[0.013]^{* *}}\end{array}$ & $\begin{array}{l}1.872 \\
{[0.000]^{* * *}}\end{array}$ & $\begin{array}{l}0.230 \\
{[0.024]^{* * *}}\end{array}$ & $\begin{array}{l}0.259 \\
{[0.018]^{* *}}\end{array}$ \\
\hline Capital expenditures/assets & $\begin{array}{l}0.3308 \\
{[0.001]^{* * *}}\end{array}$ & $\begin{array}{c}0.3694 \\
{[0.000]^{* * *}}\end{array}$ & $\begin{array}{c}0.3900 \\
{[0.001]^{* * *}}\end{array}$ & $\begin{array}{l}0.346 \\
{[0.000]^{* * *}}\end{array}$ & $\begin{array}{l}0.375 \\
{[0.000]^{* * *}}\end{array}$ & $\begin{array}{l}0.386 \\
{[0.001]^{* * * *}}\end{array}$ \\
\hline Leverage ratio & $\begin{array}{l}0.2475 \\
{[0.000]^{* * *}}\end{array}$ & $\begin{array}{l}0.3017 \\
{[0.000]^{* * * *}}\end{array}$ & $\begin{array}{l}0.3997 \\
{[0.000]^{* * * *}}\end{array}$ & $\begin{array}{l}0.242 \\
{[0.000]^{* * *}}\end{array}$ & $\begin{array}{c}0.294 \\
{[0.000]^{* * *}}\end{array}$ & $\begin{array}{l}0.390 \\
{[0.000] * * *}\end{array}$ \\
\hline Constant & $\begin{array}{l}-0.4754 \\
{[0.000]^{* * *}}\end{array}$ & $\begin{array}{l}-0.6430 \\
{[0.000]^{* * *}}\end{array}$ & $\begin{array}{l}-0.9722 \\
{[0.000]^{* * *}}\end{array}$ & $\begin{array}{c}0.161 \\
{[0.128]}\end{array}$ & $\begin{array}{l}-0.593 \\
{[0.000] * * *}\end{array}$ & $\begin{array}{l}-0.923 \\
{[0.000]^{* * *}}\end{array}$ \\
\hline$N$ & 20,345 & 20,345 & 18,177 & 20,345 & 20,345 & 18,177 \\
\hline Year fixed effects & Yes & Yes & Yes & Yes & Yes & Yes \\
\hline$R$-squared & 0.550 & 0.514 & 0.534 & 0.553 & 0.517 & 0.538 \\
\hline Adjusted $R$-squared & 0.548 & 0.514 & 0.533 & 0.551 & 0.517 & 0.538 \\
\hline
\end{tabular}

Note: $P$-values in brackets.

$* P<0.10, * P<0.05, * * P<0.01 "$ 


\subsection{Financial Leverage}

In our analyses, we have shown that the relationship between managerial performance incentives and firm risk weakens during economic downturns (and vice versa). However, one may argue that during recessions, as stock values decline, there will be a mechanical increase in financial leverage. Higher leverage would in turn lead to an automatic increase in stock return volatility.

Even though the article studies how the sensitivity of equity risk to pay-for-performance incentives vary with macroeconomic conditions, and not how equity risk varies with macroeconomic conditions, the effect of financial leverage on equity risk might also vary with factors related to pay-for-performance sensitivity. To address this concern, we estimate our main regressions (Equation (2)) separately for firms that are in the lowest quartile of the leverage distribution in our sample. ${ }^{8}$ These firms have very low financial leverage ratios: the mean leverage ratio for the firms in the lowest quartile is 0.003 , and the median leverage ratio is zero. A confirmation of our findings for the sample of very low leverage firms would alleviate the concern that the results we present are merely related to the increases (decreases) in financial leverage during economic recessions (expansions). We present the results in Table X. Our results remain unchanged for the sample of low-leverage firms.

\section{Conclusion}

In this article, we show that the relationship between performance incentives and firm risk is sensitive to the underlying macroeconomic environment. Our results suggest that the same manager with exactly the same level of performance incentives facing the same firm characteristics may target a lower (higher) risk level during economic recessions (expansions). To our knowledge, we provide the first evidence of the pro-cyclicality of this relationship, a result that is consistent with the state-dependent nature of individual risk aversion (Guiso, Sapienza, and Zingales (2014); Cohn et al., 2015).

Understanding how similar pay packages are associated with different risk levels under different economic conditions is crucial for designing compensation packages that yield a desired level of firm risk over the business cycle. A large set of corporate finance studies argues that excessive risk aversion on the part of CEOs and other senior executives is one of the most important and potentially most costly agency problems. In theory, shareholders and boards, who are well aware of the significance of this agency issue, can design contracts to mitigate executives' risk aversion. However, in practice, the contracts designed by the boards may not always sufficiently incentivize the executives to offset the effect of increasing risk aversion during recessions. Equally, not considering the effect of lower risk aversion levels during economic booms might result in managers taking excessive risks, as evidenced in the last financial crisis.

Our research highlights the importance of the interaction between managerial incentives and the macroeconomic environment. Boards and regulators, who design compensation structure to curb excessive risk taking, may find it useful to consider the pro-cyclical nature of the relationship between performance incentives and risk taking. Our results indicate that counteracting the pro-cyclical relationship between pay-for-performance incentives

8 We thank the referee for this suggestion. 
Table X. Regressions for a subsample of low-leverage firms

This table presents the results for the estimation of Equation (2) in the text using a sub-sample of low-leverage firms, that is, the firms that are in the lowest quartile of leverage distribution. The dependent variable is firm risk, calculated as the logarithm of the annualized variance of the daily stock returns. The main variable of interest is the interaction of logarithm of the lagged value of delta with the recession measure. The definitions of the variables are provided in Appendix A. All regressions control for year fixed effects. Robust standard errors are clustered at the firm level. $P$-values are provided in brackets. ${ }^{*}{ }^{* *}$, and ${ }^{* *}$ mark the $10 \%, 5 \%$, and $1 \%$ statistical significance for the estimated coefficients.

\begin{tabular}{|c|c|c|c|c|c|c|}
\hline \multirow{2}{*}{$\begin{array}{l}\text { Macroeconomic } \\
\text { state measured by: }\end{array}$} & \multicolumn{3}{|c|}{ NBER recessions } & \multicolumn{3}{|l|}{ GDP growth } \\
\hline & $\begin{array}{l}\text { Industry } \\
\text { fixed } \\
\text { effects }\end{array}$ & $\begin{array}{l}\text { Firm } \\
\text { fixed } \\
\text { effects }\end{array}$ & $\begin{array}{l}\text { Firm- } \\
\text { manager } \\
\text { fixed effects }\end{array}$ & $\begin{array}{l}\text { Industry } \\
\text { fixed } \\
\text { effects }\end{array}$ & $\begin{array}{l}\text { Firm } \\
\text { fixed } \\
\text { effects }\end{array}$ & $\begin{array}{l}\text { Firm- } \\
\text { manager } \\
\text { fixed effects }\end{array}$ \\
\hline $\log \left(\right.$ Delta $\left._{-1}\right)$ & $\begin{array}{c}0.007 \\
{[0.417]}\end{array}$ & $\begin{array}{c}0.015 \\
{[0.082]^{*}}\end{array}$ & $\begin{array}{l}0.033 \\
{[0.001]^{* * *}}\end{array}$ & $\begin{array}{r}-0.012 \\
{[0.160]}\end{array}$ & $\begin{array}{c}0.002 \\
{[0.870]}\end{array}$ & $\begin{array}{c}0.023 \\
{[0.052]^{*}}\end{array}$ \\
\hline $\log \left(\operatorname{Vega}_{-1}\right)$ & $\begin{array}{c}-0.007 \\
{[0.547]}\end{array}$ & $\begin{array}{l}-0.027 \\
{[0.013]^{* *}}\end{array}$ & $\begin{array}{l}-0.043 \\
{[0.001] * *}\end{array}$ & $\begin{array}{c}-0.013 \\
{[0.201]}\end{array}$ & $\begin{array}{l}-0.030 \\
{[0.010] * * *}\end{array}$ & $\begin{array}{l}-0.041 \\
{[0.005] * *}\end{array}$ \\
\hline Macroeconomy & $\begin{array}{l}0.268 \\
{[0.009]^{* * *}}\end{array}$ & $\begin{array}{l}0.263 \\
{[0.006]^{* * *}}\end{array}$ & $\begin{array}{c}0.233 \\
{[0.065]^{*}}\end{array}$ & $\begin{array}{l}-0.110 \\
{[0.000]^{* * *}}\end{array}$ & $\begin{array}{l}-0.116 \\
{[0.000]^{* * *}}\end{array}$ & $\begin{array}{l}-0.106 \\
{[0.000]^{* * *}}\end{array}$ \\
\hline $\begin{array}{l}\log \left(\text { Delta }_{-1}\right) * \\
\quad \text { Macroeconomic state }\end{array}$ & $\begin{array}{l}-0.027 \\
{[0.056]^{*}}\end{array}$ & $\begin{array}{l}-0.024 \\
{[0.075]^{*}}\end{array}$ & $\begin{array}{c}-0.019 \\
{[0.267]}\end{array}$ & $\begin{array}{l}0.010 \\
{[0.000]^{* * *}}\end{array}$ & $\begin{array}{l}0.009 \\
{[0.000]^{* * *}}\end{array}$ & $\begin{array}{l}0.008 \\
{[0.018]^{* *}}\end{array}$ \\
\hline $\begin{array}{l}\log \left(\operatorname{Vega}_{-1}\right) * \\
\quad \text { Macroeconomic state }\end{array}$ & $\begin{array}{l}-0.028 \\
{[0.037]^{*} *}\end{array}$ & $\begin{array}{c}-0.009 \\
{[0.483]}\end{array}$ & $\begin{array}{c}0.005 \\
{[0.733]}\end{array}$ & $\begin{array}{l}0.005 \\
{[0.032]^{* *}}\end{array}$ & $\begin{array}{c}0.002 \\
{[0.572]}\end{array}$ & $\begin{array}{r}-0.002 \\
{[0.524]}\end{array}$ \\
\hline $\log \left(\right.$ Cash compensation $\left._{-1}\right)$ & $\begin{array}{l}-0.079 \\
{[0.000]^{* * *}}\end{array}$ & $\begin{array}{l}-0.047 \\
{[0.088]^{*}}\end{array}$ & $\begin{array}{r}-0.041 \\
{[0.173]}\end{array}$ & $\begin{array}{l}-0.090 \\
{[0.000]^{* * *}}\end{array}$ & $\begin{array}{l}-0.051 \\
{[0.059]^{*}}\end{array}$ & $\begin{array}{c}-0.043 \\
{[0.151]}\end{array}$ \\
\hline CEO tenure & $\begin{array}{l}-0.009 \\
{[0.000] * * *}\end{array}$ & $\begin{array}{r}-0.003 \\
{[0.258]}\end{array}$ & $\begin{array}{c}0.002 \\
{[0.916]}\end{array}$ & $\begin{array}{l}-0.008 \\
{[0.000]^{* * *}}\end{array}$ & $\begin{array}{c}-0.003 \\
{[0.303]}\end{array}$ & $\begin{array}{c}-0.001 \\
{[0.967]}\end{array}$ \\
\hline $\log ($ Sales $)$ & $\begin{array}{l}-0.177 \\
{[0.000] * *}\end{array}$ & $\begin{array}{l}-0.132 \\
{[0.000] * *}\end{array}$ & $\begin{array}{l}-0.085 \\
{[0.017]^{* *}}\end{array}$ & $\begin{array}{l}-0.172 \\
{[0.000]^{* * *}}\end{array}$ & $\begin{array}{l}-0.124 \\
{[0.000]^{* * *}}\end{array}$ & $\begin{array}{l}-0.078 \\
{[0.026] *}\end{array}$ \\
\hline Market-to-book & $\begin{array}{c}0.005 \\
{[0.513]}\end{array}$ & $\begin{array}{c}-0.011 \\
{[0.119]}\end{array}$ & $\begin{array}{c}-0.008 \\
{[0.332]}\end{array}$ & $\begin{array}{c}0.003 \\
{[0.734]}\end{array}$ & $\begin{array}{c}-0.010 \\
{[0.156]}\end{array}$ & $\begin{array}{c}-0.007 \\
{[0.426]}\end{array}$ \\
\hline R\&D expenditures/assets & $\begin{array}{l}1.227 \\
{[0.000] * * *}\end{array}$ & $\begin{array}{l}0.459 \\
{[0.005]^{* * *}}\end{array}$ & $\begin{array}{l}0.520 \\
{[0.032]^{* *}}\end{array}$ & $\begin{array}{l}1.349 \\
{[0.000]^{* * *}}\end{array}$ & $\begin{array}{l}0.460 \\
{[0.004]^{* * *}}\end{array}$ & $\begin{array}{l}0.529 \\
{[0.025]^{* *}}\end{array}$ \\
\hline Capital expenditures/assets & $\begin{array}{l}0.727 \\
{[0.010]^{* * *}}\end{array}$ & $\begin{array}{l}0.823 \\
{[0.002]^{* * *}}\end{array}$ & $\begin{array}{l}0.809 \\
{[0.007] * * *}\end{array}$ & $\begin{array}{c}0.717 \\
{[0.012]^{* *}}\end{array}$ & $\begin{array}{l}0.825 \\
{[0.002]^{* * *}}\end{array}$ & $\begin{array}{l}0.794 \\
{[0.008]^{* * *}}\end{array}$ \\
\hline Debt/assets & $\begin{array}{c}-0.080 \\
{[0.881]}\end{array}$ & $\begin{array}{c}0.964 \\
{[0.092]^{*}}\end{array}$ & $\begin{array}{c}0.720 \\
{[0.288]}\end{array}$ & $\begin{array}{c}-0.286 \\
{[0.610]}\end{array}$ & $\begin{array}{c}0.882 \\
{[0.119]}\end{array}$ & $\begin{array}{c}0.683 \\
{[0.300]}\end{array}$ \\
\hline Constant & $\begin{array}{l}-0.798 \\
{[0.004]^{* * *}}\end{array}$ & $\begin{array}{l}-0.587 \\
{[0.002]^{* * *}}\end{array}$ & $\begin{array}{l}-0.860 \\
{[0.000]^{* * *}}\end{array}$ & $\begin{array}{l}-0.536 \\
{[0.042]^{* *}}\end{array}$ & $\begin{array}{l}-0.428 \\
{[0.028]^{* *}}\end{array}$ & $\begin{array}{l}-0.725 \\
{[0.001]^{* * *}}\end{array}$ \\
\hline$N$ & 4,930 & 4,930 & 4,443 & 4,930 & 4,930 & 4,443 \\
\hline Year fixed effects & Yes & Yes & Yes & Yes & Yes & Yes \\
\hline$R$-squared & 0.577 & 0.540 & 0.524 & 0.582 & 0.547 & 0.532 \\
\hline Adjusted $R$-squared & 0.570 & 0.537 & 0.521 & 0.575 & 0.545 & 0.529 \\
\hline
\end{tabular}

Note: $P$-values in brackets.

$* P<0.10, * * P<0.05$. 
and firm risk by providing less (more) risk-taking incentives during economic expansions (recessions) can be beneficial.

\section{References}

Agrawal, A. and Nasser, T. (2012) Blockholders on boards and CEO compensation, turnover and firm valuation, CELS 2009 4th Annual Conference on Empirical Legal Studies Paper.

Amihud, Y. and Lev, B. (1981) Risk reduction as a managerial motive for conglomerate mergers, Bell Journal of Economics 12, 605-617.

Andersen, T., Bollerslev, T., Diebold, F., and Vega, C. (2007) Real-time price discovery in stock, bond and foreign exchange markets, Journal of International Economics 73, 251-277.

Armstrong, C. and Vashishtha, R. (2012) Executive stock options, differential risk-taking incentives, and firm value Journal of Financial Economics 104, 70-88.

Baker, M. and Gompers, P. A. (2003) The determinants of board structure at the initial public offering, Journal of Law and Economics 46, 569-598.

Barker, V. L., III and Mueller G. C. (2002) CEO characteristics and firm R\&D spending, Management Science 48, 782-801.

Bebchuk, L. A., Grinstein, Y., and Peyer, U. (2010) Lucky CEOs and lucky directors, Journal of Finance 65, 2363-2401.

Bizjak, J., Brickley, J., and Coles, J. (1993) Stock-based incentive compensation and investment behavior, Journal of Accounting and Economics 16, 349-372.

Black, F. and Scholes, M. (1973) The pricing of options and corporate liabilities, Journal of Political Economy 81, 637-654.

Bloom, N. and Van Reenen, J. (2007) Measuring and explaining management practices across firms and countries, Quarterly Journal of Economics 122, 1351-1408.

Bloom, N., Propper, C., Seiler, S., and Van Reenen, J. (2015) The impact of competition on management quality: Evidence from public hospitals, Review of Economic Studies 82, 457-489.

Boyd, J., Jagannathan, R., and Hu, J. (2005) The stock market's reaction to unemployment news: Why bad news is usually good for stocks, Journal of Finance 60, 649-672.

Brown, K. C., Harlow, W. V., and Starks, L. T. (1996) Of tournaments and temptations: An analysis of managerial incentives in the mutual fund industry, Journal of Finance 51, 85-110.

Bun, M. and Harrison, T. (2014) OLS and IV estimation of regression models including endogenous interaction terms. Working paper, LeBow College of Business, Drexel University.

Chava, S., Kumar, P., and Warga, A. (2010) Managerial agency and bond covenants, Review of Financial Studies 23, 1120-1148.

Chhaochharia, V., Grinstein, Y., Grullon, G., and Michaely, R. (2012) Product market competition and internal governance: Evidence from the Sarbanes Oxley Act. Johnson School Research Paper Series (18-2012).

Cohn, A., Engelmann, J., Fehr, E., and Maréchal, M. A. (2015) Evidence for countercyclical risk aversion: An experiment with financial professionals, American Economic Review 105, 860-885.

Coles, J., Daniel, N., and Naveen, L. (2006) Managerial incentives and risk-taking, Journal of Financial Economics 79, 431-468.

Coles, J. L., Daniel, N., and Naveen, L. (2014) Co-opted boards, Review of Financial Studies 27, 1751-1796.

Core, J. and Guay, W. (1999) The use of equity grants to manage optimal equity incentive levels, Journal of Accounting \& Economics 28, 151-184.

Core, J. and Guay, W. (2002) Estimating the value of employee stock option portfolios and their sensitivities to price and volatility, Journal of Accounting Research 40, 613-630. 
Davis, S. J. and von Wachter, T. (2011) Recessions and the costs of job loss. Brookings Papers on Economic Activity Fall, 1-72.

DeYoung, R., Peng, E. M., and Yan, M. (2013) Executive compensation and business policy choices at U.S. commercial banks, Journal of Financial and Quantitative Analysis 48, 165-196.

Dittmann, I. and Yu, K. (2011) How important are risk-taking incentives in executive compensation?. Working paper, Tinbergen Institute.

Edmans, A. and Gabaix, X. (2011) The effect of risk on the CEO market, Review of Financial Studies 24, 2822-2863.

Fahlenbrach, R. (2009) Shareholder rights, boards, and CEO compensation, Review of Finance 13, 81-113.

Farber, H. (2011) Job loss in the great recession: Historical perspective from the displaced workers survey, 1984-2010. Working paper 17040, National Bureau of Economic Research.

Ferreira, D., Ferreira, M. A., and Raposo, C. C. (2011) Board structure and price informativeness, Journal of Financial Economics 99, 523-545.

Flannery, M. and Protopapadakis, A. (2002) Macroeconomic factors do influence aggregate stock returns, Review of Financial Studies 15, 751-782.

Gaver, J. and Gaver, K. (1993) Additional evidence on the association between the investment opportunity set and corporate financing, dividend, and compensation policies, Journal of Accounting and Economics 16, 125-160.

Giroud, X. and Mueller, H. (2010) Does corporate governance matter in competitive industries?, Journal of Financial Economics 95, 312-331.

Giroud, X., and Mueller, H. M. (2011) Corporate governance, product market competition, and equity prices, The Journal of Finance, 66, 563-600.

Graham, J., Harvey, C., and Puri, M. (2013) Managerial attitudes and corporate actions, Journal of Financial Economics 109, 103-121.

Greene, W. (2003) Econometric Analysis, 5th edition, Prentice Hall, Upper Saddle River, New Jersey.

Grullon, G. and Michaely, R. (2012) The impact of product market competition on firms' payout policy, Working paper, Rice University.

Guay, W. (1999) The sensitivity of CEO wealth to equity risk: An analysis of the magnitude and determinants, Journal of Financial Economics 53, 43-71.

Guiso, L., Sapienza, P., and Zingales, L. (2014). Time varying risk aversion. Available at http://fac ulty.chicagobooth.edu/luigi.zingales/papers/research/time_varying_risk_aversion.pdf.

Guvenen, F., Ozkan, S., and Song, J. (2014) The nature of countercyclical income risk, Journal of Political Economy 122, 621-660.

Hansen, L. P. (1982) Large sample properties of generalized method of moments estimators, Econometrica 50, 1029-1054.

Haugen, R. and Senbet, L. (1981) Resolving the agency problem of external capital through options, Journal of Finance 36, 629-647.

Hayes, R., Lemmon, M., and Qiu, M. (2012) Stock options and managerial incentives for risk-taking: Evidence from FAS 123R, Journal of Financial Economics 105, 174-190.

Hoberg, G. and Phillips, G. (2014) Text-based network industries and endogenous product differentiation. Working paper, University of Southern California.

Hoberg, G., Phillips, G., and Prabhala, N. (2014) Product market threats, payouts, and financial flexibility, Journal of Finance 69, 293-324.

Hong, H. and Kacperczyk, M. (2009) The price of sin: The effects of social norms on markets, Journal of Financial Economics 93, 15-36.

Jensen, M. and Meckling, W. (1976) Rights and production functions: An application to labormanaged firms and codetermination, Journal of Business 52, 469-506. 
Ju, N., Leland, H., and Senbet, L. (2002) Options, option repricing and severance packages in managerial compensation: Their effects on corporate risk. Working paper, University of Maryland and University of California, Berkeley.

Kempf, A., Ruenzi, S., and Thiele, T. (2009) Employment risk, compensation incentives, and managerial risk taking: Evidence from the mutual fund industry, Journal of Financial Economics 92, 92-108.

Low, A. (2009) Managerial risk-taking behavior and equity-based compensation, Journal of Financial Economics 92, 470-490.

Malmendier, U. and Nagel, S. (2011) Depression babies: Do macroeconomic experiences affect risk-taking?, Quarterly Journal of Economics 126, 373-416.

Masulis, R. W., Wang, C., and Xie, F. (2007) Corporate governance and acquirer returns, Journal of Finance 62, 1851-1889.

McQueen, G. and Roley, V. V. (1993) Stock prices, news and business conditions, Review of Financial Studies 92, 307-328.

Milidonis, A. and Stathopoulos, K. (2014) Managerial incentives, risk aversion and debt, Journal of Financial and Quantitative Analysis 1-61.

Morse, A., Nanda, V., and Seru, A. (2011) Are incentive contracts rigged by powerful CEOs?, Journal of Finance 66, 1779-1821.

Murphy, K. (2012) Executive compensation: Where we are, and how we got there, in: G. Constantinides, M. Harris, and R. Stulz (eds.), Handbook of the Economics of Finance, Elsevier Science, North Holland.

Myers, S. (1977) Determinants of corporate borrowing, Journal of Financial Economics 5, $147-175$.

Neyland, J. (2012) Wealth shocks and executive compensation: Evidence from CEO divorce. Available at SSRN 2140668.

Ou-Yang, H. (2003) Optimal contracts in a continuous? Time delegated portfolio management problem, Review of Financial Studies 16, 173-208.

Pan, Y., Wang, T. Y., and Weisbach, M. S. (2014) CEO investment cycles. Working paper, Fisher College of Business.

Petersen, M. A. (2009) Estimating standard errors in finance panel data sets: Comparing approaches, Review of Financial Studies 22, 435-480.

Savaser, T. and Şişli-Ciamarra, E. (2014) Managerial risk-taking incentives and firm risk in the post-regulatory era. Working paper, Brandeis University and Bilkent University.

Schoar, A. and Zuo, L. (2013) Shaped by booms and busts: How the economy impacts CEO careers and management styles. Working paper 17590, National Bureau of Economic Research.

Schmidt, P. (1976) Econometrics. Marcel Dekker, Inc., New York.

Shivdasani, A. and Yermack, D. (1999) CEO involvement in the selection of new board members: An empirical analysis, Journal of Finance 54, 1829-1853.

Smith, C. and Stulz, R. (1985) The determinants of firms' hedging policies, Journal of Financial and Quantitative Analysis 20,391-405.

Smith, C. and Watts, R. (1992) The investment opportunity set and corporate financing, dividend and compensation policy, Journal of Financial Economics 3, 263-292.

Thompson, S. B. (2011) Simple formulas for standard errors that cluster by both firm and time, Journal of Financial Economics 99, 1-10.

Tufano, P. (1996) Who manages risk? An empirical examination of risk management practices in the gold mining industry, Journal of Finance 51, 1097-1137.

Wooldridge, J. (2002) Econometric Analysis of Cross Section and Panel Data, the MIT Press, Cambridge, Massachusetts. 
Appendix A.

Table Al. Variable definitions and sources

\begin{tabular}{|c|c|c|}
\hline Variable & Description & Source \\
\hline \multicolumn{3}{|l|}{ A. CEO incentive measures } \\
\hline Salary $(\$ 000 s)$ & Base salary of the CEO & ExecuComp \\
\hline Bonus ( $\$ 000 \mathrm{~s})$ & $\begin{array}{l}\text { Bonus payments to the CEO. Calculated } \\
\text { as "Bonus + Non-equity Incentives" } \\
\text { after the fiscal year } 2006\end{array}$ & ExecuComp \\
\hline Cash compensation $(\$ 000)$ & Salary plus bonus & ExecuComp \\
\hline Delta (\$000s) & $\begin{array}{l}\text { Dollar change in the CEO stock and op- } \\
\text { tion portfolio for a } 1 \% \text { change in } \\
\text { stock price }\end{array}$ & Authors' calculations \\
\hline Vega $(\$ 000 s)$ & $\begin{array}{l}\text { Dollar change in the CEO stock and op- } \\
\text { tion portfolio for a } 1 \% \text { change in } \\
\text { stock return volatility }\end{array}$ & Authors' calculations \\
\hline Tenure as CEO (years) & Number of years as CEO & ExecuComp \\
\hline Long tenure & $\begin{array}{c}\text { An indicator variable that takes the } \\
\text { value one if CEO tenure is greater } \\
\text { than } 3 \text { years, and zero otherwise }\end{array}$ & ExecuComp \\
\hline \multicolumn{3}{|l|}{ B. Risk measures } \\
\hline Total risk & $\begin{array}{l}\text { Annualized variance of daily stock re- } \\
\text { turns during a firm's fiscal year }\end{array}$ & CRSP \\
\hline Firm-specific risk & $\begin{array}{l}\text { Annualized variance of residuals from } \\
\text { the market model }\end{array}$ & CRSP \\
\hline \multicolumn{3}{|l|}{ C. Firm financial characteristics } \\
\hline R\&D expenditures/assets & $\begin{array}{l}\text { Research and development expenditures } \\
\text { scaled by total assets }\end{array}$ & Compustat \\
\hline Capital expenditures/assets & $\begin{array}{l}\text { Capital expenditures net of sales of } \\
\text { plant, property, and equipment scaled } \\
\text { by total assets }\end{array}$ & Compustat \\
\hline Leverage ratio & $\begin{array}{l}\text { Ratio of long-term debt and debt in cur- } \\
\text { rent liabilities to book value of assets }\end{array}$ & Compustat \\
\hline Size & Natural logarithm of net sales & Compustat \\
\hline Market value & $\begin{array}{l}\text { Sum of market value of common stock, } \\
\text { liquidating value of preferred stock, } \\
\text { and book value of total debt }\end{array}$ & Compustat, CRSP \\
\hline Market-to-book ratio & $\begin{array}{l}\text { Market value divided by book value of } \\
\text { total assets }\end{array}$ & Compustat \\
\hline Return on assets (ROA) & $\begin{array}{l}\text { Earnings before interest, taxes, depreci- } \\
\text { ation, and amortization (EBITDA) } \\
\text { scaled by total assets }\end{array}$ & Compustat \\
\hline Stock return & $\begin{array}{l}\text { Annual stock return over a firm's fiscal } \\
\text { year }\end{array}$ & Compustat \\
\hline FAS cost & $\begin{array}{l}\text { Ratio of Black-Scholes value of CEO } \\
\text { stock option grants to net income. } \\
\text { This variable serves as a proxy for ac- } \\
\text { counting cost associated with the im- } \\
\text { plementation of FAS } 123 \mathrm{R}\end{array}$ & Compustat \\
\hline
\end{tabular}


Table Al. Continued

\begin{tabular}{|c|c|c|}
\hline Variable & Description & Source \\
\hline Surplus cash & $\begin{array}{l}\text { Ratio of cash from assets-in-place (oper- } \\
\text { ating activities net cash flow minus de- } \\
\text { preciation plus R\&D expenditures) to } \\
\text { total assets }\end{array}$ & Compustat \\
\hline Total loss carry forward & $\begin{array}{l}\text { An indicator variable that takes the } \\
\text { value one if a firm has tax-loss-carry- } \\
\text { forwards in any of the past } 3 \text { years } \\
\text { and zero otherwise }\end{array}$ & Compustat \\
\hline Industry median delta & $\begin{array}{l}\text { The median value of delta in a firm's in- } \\
\text { dustry as identified by two-digit SIC } \\
\text { codes }\end{array}$ & Execucomp \\
\hline Industry median vega & $\begin{array}{l}\text { The median value of vega in a firm's in- } \\
\text { dustry as identified by two-digit SIC } \\
\text { codes }\end{array}$ & ExecuComp \\
\hline Value decline & $\begin{array}{l}\text { An indicator variable that takes the } \\
\text { value one if a firm has a negative hold- } \\
\text { ing period return in a fiscal year, and } \\
\text { zero otherwise }\end{array}$ & CRSP \\
\hline HHI & $\mathrm{HHI}$ of industry concentration & $\begin{array}{l}\text { Hoberg and Phillips } \\
\text { Data Library }\end{array}$ \\
\hline \multicolumn{3}{|l|}{ D. Macroeconomic risk measures } \\
\hline Recession dummy & $\begin{array}{l}\text { An indicator variable that takes the } \\
\text { value one if a firm's fiscal year corres- } \\
\text { ponds with } 90 \text { recession days as iden- } \\
\text { tified by the National Bureau of } \\
\text { Economic Research (NBER) and zero } \\
\text { otherwise }\end{array}$ & NBER \\
\hline Recession days & $\begin{array}{l}\text { The number of days a firm faced reces- } \\
\text { sion over a fiscal year }\end{array}$ & NBER \\
\hline $\begin{array}{l}\text { Gross domestic product } \\
\text { (GDP) growth rate }\end{array}$ & $\begin{array}{l}\text { Advance release values for real GDP } \\
\text { growth rate (percentage changes from } \\
\text { a year ago), seasonally adjusted. We } \\
\text { calculate the minimum GDP growth } \\
\text { rate over a fiscal year as the GDP } \\
\text { measure }\end{array}$ & $\begin{array}{l}\text { Bureau of Economic } \\
\text { Analysis/Action } \\
\text { Economics }\end{array}$ \\
\hline $\begin{array}{l}\text { Additions to non-farm pay- } \\
\text { roll employment (NFP) }\end{array}$ & $\begin{array}{l}\text { Advance release values for the change in } \\
\text { the non-farm payroll, seasonally ad- } \\
\text { justed. We calculate the minimum of } \\
\text { the changes in the non-farm payroll } \\
\text { over a fiscal year as the NFP measure }\end{array}$ & $\begin{array}{l}\text { Bureau of Labor } \\
\text { Statistics/Action } \\
\text { Economics }\end{array}$ \\
\hline $\begin{array}{l}\text { Personal expenditure }(\mathrm{PE}) \\
\text { growth rate }\end{array}$ & $\begin{array}{l}\text { Advance release values of the Personal } \\
\text { Consumption Expenditure Growth, } \\
\text { seasonally adjusted real values. We } \\
\text { calculate the minimum of the personal } \\
\text { expenditure growth rate over a fiscal } \\
\text { year as the PCE measure }\end{array}$ & $\begin{array}{l}\text { Bureau of Economic } \\
\text { Analysis/Action } \\
\text { Economics }\end{array}$ \\
\hline
\end{tabular}


Table Al. Continued

\begin{tabular}{lll}
\hline Variable & Description & Source \\
\hline Retail sales (RS) growth rate & $\begin{array}{c}\text { Advance release values of growth in the } \\
\text { total receipts at stores that sell mer- } \\
\text { chandise and related services to final } \\
\text { consumers. We calculate the min- } \\
\text { imum of the personal expenditure } \\
\text { growth rate over a fiscal year as the }\end{array}$ & $\begin{array}{c}\text { US Census Bureau/ } \\
\text { Action Economics }\end{array}$ \\
& $\begin{array}{l}\text { PCE measure } \\
\text { Volatility of the S\&P 500 Index during a } \\
\text { S\&P500 volatility }\end{array}$ & CRSP \\
& firm's fiscal year & \\
\hline
\end{tabular}

